ESAIM: M2AN 47 (2013) 125-147

DOI: $10.1051 / \mathrm{m} 2 \mathrm{an} / 2012023$
ESAIM: Mathematical Modelling and Numerical Analysis

www.esaim-m2an.org

\title{
UNIFORM CONVERGENCE OF LOCAL MULTIGRID METHODS FOR THE TIME-HARMONIC MAXWELL EQUATION*
}

\author{
Huangxin Chen $^{1}$, Ronald H.W. Hoppe ${ }^{2,3}$ And Xuejun Xu ${ }^{4}$
}

\begin{abstract}
For the efficient numerical solution of indefinite linear systems arising from curl conforming edge element approximations of the time-harmonic Maxwell equation, we consider local multigrid methods (LMM) on adaptively refined meshes. The edge element discretization is done by the lowest order edge elements of Nédélec's first family. The LMM features local hybrid Hiptmair smoothers of Jacobi and Gauss-Seidel type which are performed only on basis functions associated with newly created edges/nodal points or those edges/nodal points where the support of the corresponding basis function has changed during the refinement process. The adaptive mesh refinement is based on Dörfler marking for residual-type a posteriori error estimators and the newest vertex bisection strategy. Using the abstract Schwarz theory of multilevel iterative schemes, quasi-optimal convergence of the LMM is shown, i.e., the convergence rates are independent of mesh sizes and mesh levels provided the coarsest mesh is chosen sufficiently fine. The theoretical findings are illustrated by the results of some numerical examples.
\end{abstract}

Mathematics Subject Classification. 65N30, 65N50, 65N55, 78M60.

Received June 28, 2011. Revised December 6, 2011.

Published online July 31, 2012.

\section{INTRODUCTION}

In this paper, we develop, analyze, and implement local multigrid methods for indefinite algebraic systems arising from adaptive curl-conforming edge element approximations of the time-harmonic Maxwell equation. In particular, we consider a lossless medium occupying a bounded Lipschitz polyhedron $\Omega \subset \mathbb{R}^{3}$ with a perfectly conducting boundary $\partial \Omega$. Given a solenoidal current density $\boldsymbol{f}$, the problem is to compute a time-harmonic

Keywords and phrases. Maxwell equations, Nédélec edge elements, indefinite, multigrid methods, local Hiptmair smoothers, adaptive edge finite element methods, optimality.

* The second author acknowledges support by NSF DMS-0810176 and also expresses his sincere thanks to the Institute for Mathematics and its Applications (IMA) at the University of Minnesota, Minneapolis, for its kind hospitality during his stay in Fall 2010. The work of the third author was supported by the National Basis Research Projects (2005CB321701 and 2011CB30971) and the National Science Foundation of China (10731060 and 11171335).

1 LSEC, Institute of Computational Mathematics and Scientific/Engineering Computing, Academy of Mathematics and System Sciences, Chinese Academy of Sciences, P.O. Box 2719, Beijing 100190, P.R. China. chx@lsec.cc.ac.cn

2 Department of Mathematics, University of Houston, Houston, 77204-3008 TX, USA. rohop@math.uh.edu

3 Institute for Mathematics, University of Augsburg, 86159 Augsburg, Germany.

4 LSEC, Institute of Computational Mathematics and Scientific/Engineering Computing, Academy of Mathematics and System Sciences, Chinese Academy of Sciences, P.O. Box 2719, Beijing 100190, P.R. China. xxj@lsec.cc.ac.cn 
electric field $\boldsymbol{u}$ in $\Omega$ with wave number $\kappa>0$ such that

$$
\begin{array}{rlrl}
\operatorname{curl} \operatorname{curl} \boldsymbol{u}-\kappa^{2} \boldsymbol{u} & =\boldsymbol{f} & & \text { in } \Omega, \\
\boldsymbol{u} \times \boldsymbol{n}=\mathbf{0} & & \text { on } \partial \Omega,
\end{array}
$$

where $\boldsymbol{n}$ stands for the unit outward normal on $\partial \Omega$. The choice of the boundary condition (1.1b) is made for ease of presentation only. Under the assumption that $\kappa^{2}$ is not a Maxwell eigenvalue, i.e., $\kappa^{2}$ is not an eigenvalue of the curl-curl operator, it is well-known that (1.1a), (1.1b) has a unique solution (cf. e.g., [27], [29], Chap. 4).

Curl-conforming edge elements, originally known as Whitney forms [36] and designed to study the multiplicity of zero as an eigenvalue of the Hodge Laplacian, have been introduced to the numerical analysis community by Nédélec $[30,31]$ and since then have become a standard tool in computational electromagnetism $(c f .[8,21,29]$ and the references therein). An intrinsic difficulty in the numerical solution of edge element discretized PDEs involving the curl-curl operator is the non-trivial kernel of the discrete curl operator which is given by the gradients of the standard nodal basis functions. Within the multigrid iterative solution, this has been taken care of by hybrid smoothers, namely the Hiptmair smoother [20] and the Arnold-Falk-Winther smoother [3] which have been originally designed for $\boldsymbol{H}(\mathbf{c u r l} ; \Omega)$-elliptic problems. Adaptive edge finite element methods (AEFEM) on the basis of residual-type a posteriori error estimators have been developed first in $[6,7,28]$ and further studied in $[12,15,25,41]$. Quasi-optimal convergence of AEFEM for the time-harmonic Maxwell equations has been established recently in [42].

In this paper, we are interested in local multigrid methods (LMM) on adaptively refined meshes obtained from the application of AEFEM to the time-harmonic Maxwell equation (1.1a),(1.1b) and to prove uniform convergence of the LMM which together with [42] results in an overall quasi-optimal algorithm. LMM on adaptively refined meshes feature hybrid smoothing only on new edges/nodes and those old edges/nodes where the support of the associated edge/nodal basis function has changed. This strategy makes the computational cost on each level of the LMM proportional to the number of elements appearing in the local refinement. The idea can be traced back to multilevel adaptive techniques (MLAT) studied in $[4,10,32]$ and multigrid methods for locally refined finite element meshes $[1,2,16,33]$. However, these locally refined meshes obey restrictive conditions which are not satisfied by the newest vertex bisection algorithm ( $c f .[37,38]$ and the references therein) which will be used for adaptivity in this paper. The uniform convergence theory of LMM for $2 \mathrm{D}$ and 3D $H^{1}(\Omega)$ elliptic problems has been studied in $[24,37,39,40]$. The hierarchy of meshes used in the LMM can be obtained either by successive adaptive refinement of an initial coarse mesh or by successive coarsening of a fine mesh. Recently, Hiptmair et al. [23, 24], and Xu et al. [38] have developed LMM based on a different strategy for the construction of hierarchies of meshes and have succeeded to establish uniform convergence in case of $\boldsymbol{H}(\mathbf{c u r l} ; \Omega)$ elliptic problems. We emphasize that in our algorithms we do not reconstruct a virtual refinement hierarchy of meshes, but use the hierarchy generated by the AEFEM. For time-harmonic Maxwell problems, LMM with hybrid smoothers have been studied numerically in $[6,14,26,34]$. The computational results in these papers indicate efficiency and robustness of the approach. But so far, there does not exist any theoretical investigation in the literature. In this paper, using the methodology developed in [13,19], we present a convergence analysis which is based on a perturbation of the estimates for $\boldsymbol{H}(\mathbf{c u r l} ; \Omega)$-elliptic problems. In our analysis, we apply the techniques from $[18,19]$ and show that LMM with additive local Hiptmair-Jacobi smoothers or multiplicative local Hiptmair-Gauss-Seidel smoothers converge uniformly provided that the coarsest grid is chosen sufficiently fine, a condition that seems to be unavoidable in the current numerical solution of time-harmonic Maxwell equations. The main difficulties in the convergence analysis are:

- how to apply the perturbation analysis and a $\boldsymbol{H}(\mathbf{c u r l} ; \Omega)$-elliptic stable multilevel decomposition of the edge element space to obtain the estimate (A1) in Section 3;

- how to apply a global strengthened Cauchy-Schwarz inequality with respect to this decomposition to get the estimate (A3);

- how to get a global spectral estimate. 
The remainder of this paper is organized as follows. In Section 2, we introduce the weak formulation and the edge finite element approximation of (1.1a), (1.1b), and address the LMM featuring additive and multiplicative local Hiptmair smoothers. The convergence theory of the LMM is developed in Section 3 within the abstract framework of the Schwarz theory of multilevel iterative schemes, whereas Section 4 is devoted to the verification of the assumptions required by the abstract theory for the local Hiptmair smoothers. In the final Section 5, we present the results of some numerical experiments illustrating the performance of the LMM and exemplifying our theoretical results.

\section{Edge ELEMENT APPROXIMATION AND LOCAL MULtigRid METHOdS}

Throughout this paper, we adopt standard notation from Lebesgue and Sobolev space theory (cf., e.g., [35]). In particular, we refer to $L^{2}(\Omega)$ and $H^{m}(\Omega), m \in \mathbb{N}$, as the Hilbert space of Lebesgue integrable functions in $\Omega$ and the Sobolev space of $L^{2}$-functions with $L^{2}$-integrable weak derivatives up to order $m$. For non-integer value $s \in \mathbb{R}_{+}$, the Sobolev space $H^{s}(\Omega)$ is defined by interpolation. Likewise, $\boldsymbol{L}^{2}(\Omega)$ and $\boldsymbol{H}^{s}(\Omega)$ stand for the corresponding Hilbert spaces of vector-valued functions. In both cases, the inner products and associated norms will be denoted by $(\cdot, \cdot)_{s, \Omega}$ and $\|\cdot\|_{s, \Omega}$, respectively. For brevity, let $(\cdot, \cdot)$ denote the $L^{2}$ inner product. For a function $v \in H^{s}(\Omega)$, we denote by $\left.v\right|_{\partial \Omega}$ the trace of $v$ on $\partial \Omega$ and define $H_{0}^{s}(\Omega):=\left\{v \in H^{s}(\Omega)|v|_{\partial \Omega}=0\right\}$. Moreover, we denote by $\boldsymbol{H}(\operatorname{curl} ; \Omega):=\left\{\boldsymbol{v} \in \boldsymbol{L}^{2}(\Omega) \mid \operatorname{curl} \boldsymbol{v} \in \boldsymbol{L}^{2}(\Omega)\right\}$ and $\boldsymbol{H}(\operatorname{div} ; \Omega):=\left\{\boldsymbol{v} \in \boldsymbol{L}^{2}(\Omega) \mid \operatorname{div} \boldsymbol{v} \in\right.$ $\left.L^{2}(\Omega)\right\}$ the Hilbert spaces of vector-valued functions with the inner products $(\cdot, \cdot)_{\operatorname{curl}, \Omega},(\cdot, \cdot)_{\operatorname{div}, \Omega}$ and associated norms $\|\cdot\|_{c u r l, \Omega},\|\cdot\|_{d i v, \Omega}$. We further refer to $\boldsymbol{H}_{0}(\operatorname{curl} ; \Omega):=\left\{\boldsymbol{v} \in \boldsymbol{H}(\boldsymbol{c u r l} ; \Omega)|(\boldsymbol{v} \times \boldsymbol{n})|_{\partial \Omega}=0\right\}$ as the subspace of vector fields with vanishing tangential trace on $\partial \Omega$ and to $\boldsymbol{H}\left(\operatorname{div}^{0} ; \Omega\right):=\{\boldsymbol{v} \in \boldsymbol{H}(\operatorname{div} ; \Omega) \mid \operatorname{div} \boldsymbol{v}=0\}$ as the subspace of solenoidal vector fields.

For a given $\boldsymbol{f} \in \boldsymbol{H}\left(\operatorname{div}^{0} ; \Omega\right)$, the weak formulation of (1.1a) and (1.1b) is to find $\boldsymbol{u} \in \boldsymbol{H}_{0}(\mathbf{c u r l} ; \Omega)$ such that

$$
\boldsymbol{a}(\boldsymbol{u}, \boldsymbol{v})=(\boldsymbol{f}, \boldsymbol{v}), \quad \boldsymbol{v} \in \boldsymbol{H}_{0}(\operatorname{curl} ; \Omega),
$$

where the bilinear form $\boldsymbol{a}: \boldsymbol{H}_{0}(\mathbf{c u r l} ; \Omega) \times \boldsymbol{H}_{0}(\operatorname{curl} ; \Omega) \rightarrow \mathbb{R}$ is given by

$$
\boldsymbol{a}(\boldsymbol{u}, \boldsymbol{v})=(\operatorname{curl} \boldsymbol{u}, \operatorname{curl} \boldsymbol{v})-\kappa^{2}(\boldsymbol{u}, \boldsymbol{v}), \quad \boldsymbol{u}, \boldsymbol{v} \in \boldsymbol{H}_{0}(\operatorname{curl} ; \Omega) .
$$

We further introduce a symmetric positive definite form $\hat{\boldsymbol{a}}(\cdot, \cdot)$ according to

$$
\hat{\boldsymbol{a}}(\boldsymbol{u}, \boldsymbol{v}):=(\boldsymbol{u}, \boldsymbol{v})_{\operatorname{curl}, \Omega}, \quad \boldsymbol{u}, \boldsymbol{v} \in \boldsymbol{H}_{0}(\operatorname{curl} ; \Omega) .
$$

For any subdomain $D \subset \Omega$, we define the associated energy norm by $\|\cdot\|_{\hat{\boldsymbol{a}}, D}^{2}:=\left.\hat{\boldsymbol{a}}(\cdot, \cdot)\right|_{D}$. We omit the subscript $D$ when $D=\Omega$.

Let $\left\{\mathcal{T}_{l}, l=0,1, \ldots, L\right\}$ be a shape regular family of nested geometrically conforming simplicial triangulations of the computational domain $\Omega$ obtained by successive refinement of a sufficiently fine initial coarse mesh $\mathcal{T}_{0}$ using newest vertex bisection. The initial mesh size is scaled such that $h_{0}<1$. We define $\mathcal{E}_{l}$ as the set of interior edges on $\mathcal{T}_{l}$ and $\mathcal{N}_{l}$ as the set of interior nodes of $\mathcal{T}_{l}$. We further refer to $\Omega_{l}^{p}$ as the union of elements in $\mathcal{T}_{l}$ containing $\boldsymbol{p} \in \mathcal{N}_{l}$ and to $\Omega_{l}^{E}$ as the union of elements in $\mathcal{T}_{l}$ containing $E \in \mathcal{E}_{l} . \mathcal{T}_{l}\left(\Omega_{l}^{p}\right) \subset \mathcal{T}_{l}$ and $\mathcal{T}_{l}\left(\Omega_{l}^{E}\right) \subset \mathcal{T}_{l}$ denote the sets of elements contained in $\Omega_{l}^{p}$ and $\Omega_{l}^{E}$, respectively. The quantities $h_{l, \boldsymbol{p}}, h_{l, E}$ stand for the diameters of the subdomains $\Omega_{l}^{p}, \Omega_{l}^{E}$, and for any tetrahedron $T \in \mathcal{T}_{l}, h_{T}$ refers to the diameter of $T$. Moreover, $\mathcal{G}(T)$ is the number of bisections needed to generate $T$ from an element in $\mathcal{T}_{0}$. It is reasonable to assume [5]

$$
C_{d} \theta^{m} \leq h_{T} \leq C_{u} \theta^{m}, \quad m=\mathcal{G}(T), \quad \forall T \in \bigcup_{l=0}^{L} \mathcal{T}_{l},
$$

where $0<\theta<1$, and $C_{d}, C_{u}$ are positive constants that only depend on $\mathcal{T}_{0}$ and the shape regularity of the meshes. Throughout this paper, \#S denotes the cardinality of a set $S$, and $C$, with or without subscript, denotes a generic positive constant. This constant, depending on the wave number $\kappa$ and the shape regularity of the 

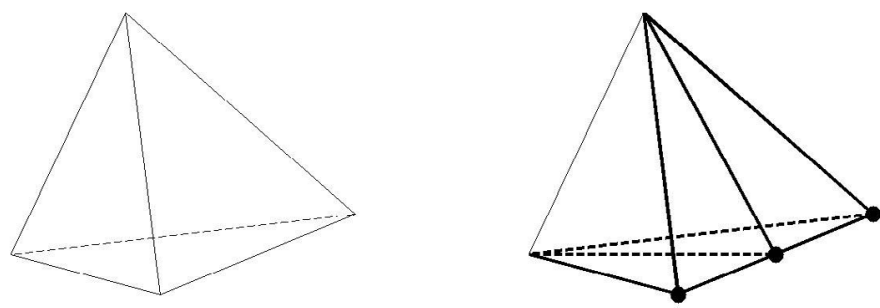

Figure 1. The left figure is a tetrahedron in $\mathcal{T}_{l-1}$ to be refined. The right figure shows that the tetrahedron is bisected into two tetrahedra in $\mathcal{T}_{l}$. The big vertices in the right figure are the local smoothing vertices contained in $\widetilde{\mathcal{N}}_{l}$, and the boldfaced edges are the local smoothing edges contained in $\widetilde{\mathcal{E}}_{l}$.

meshes, can take on different values in different occurrences but will always be independent of mesh sizes and mesh levels.

For $l=0, \ldots, L$, let $\boldsymbol{U}_{l}$ denote the curl-conforming edge element space generated by the lowest order edge elements of Nédélec's first family [30] with respect to the mesh $\mathcal{T}_{l}$. Since the meshes are nested, we have a sequence of nested edge element spaces $\boldsymbol{U}_{0} \subset \boldsymbol{U}_{1} \subset \cdots \subset \boldsymbol{U}_{L}$. The finite element approximation of (2.1) is to find $\boldsymbol{u}_{l} \in \boldsymbol{U}_{l}$ such that

$$
\boldsymbol{a}\left(\boldsymbol{u}_{l}, \boldsymbol{v}_{l}\right)=\left(\boldsymbol{f}, \boldsymbol{v}_{l}\right), \quad \boldsymbol{v}_{l} \in \boldsymbol{U}_{l} .
$$

Under the assumption that $\max _{T \in \mathcal{T}_{l}} h_{T}$ is sufficiently small, existence and uniqueness of the solution $\boldsymbol{u}_{l}$ are well-known $[21,29]$. In particular, the projector

$$
\boldsymbol{a}\left(\boldsymbol{P}_{l} \boldsymbol{v}, \boldsymbol{w}\right)=\boldsymbol{a}(\boldsymbol{v}, \boldsymbol{w}), \quad \boldsymbol{v} \in \boldsymbol{U}_{L}, \boldsymbol{w} \in \boldsymbol{U}_{l}, 0 \leq l \leq L,
$$

is well defined. We further denote by $\boldsymbol{Q}_{l}: \boldsymbol{L}^{2}(\Omega) \rightarrow \boldsymbol{U}_{l}$ the $L^{2}$-projector onto $\boldsymbol{U}_{l}$. By a similar technique as in [18], Lemma 4.3, the imbedding results in [29], Theorem 3.50 and Corollary 3.51, and the estimate in [29], Lemma 7.6, for discrete divergence-free vector fields we have the following estimate:

Lemma 2.1. Let $\Omega$ be a bounded Lipschitz polyhedral domain. If the initial mesh size $h_{0}$ is sufficiently small, there exists a constant $s \in(1 / 2,1]$, depending only on the domain $\Omega$, such that for any $\boldsymbol{v} \in \boldsymbol{U}_{L}$ and $\boldsymbol{w}_{0} \in \boldsymbol{U}_{0}$ there holds

$$
\left(\boldsymbol{v}-\boldsymbol{P}_{0} \boldsymbol{v}, \boldsymbol{w}_{0}\right) \leq C h_{0}^{s}\left\|\boldsymbol{v}-\boldsymbol{P}_{0} \boldsymbol{v}\right\|_{\hat{\boldsymbol{a}}}\left\|\boldsymbol{w}_{0}\right\|_{\hat{\boldsymbol{a}}} .
$$

For $0 \leq l \leq L$, we define the level $l$ operator $\boldsymbol{A}_{l}: \boldsymbol{U}_{l} \rightarrow \boldsymbol{U}_{l}$ according to

$$
\left(\boldsymbol{A}_{l} \boldsymbol{v}, \boldsymbol{w}\right)=\boldsymbol{a}(\boldsymbol{v}, \boldsymbol{w}), \quad \boldsymbol{v}, \boldsymbol{w} \in \boldsymbol{U}_{l} .
$$

and refer to $\boldsymbol{f}_{l} \in \boldsymbol{U}_{l}$ as the $\mathbf{L}^{2}$-projection of $\boldsymbol{f}$ onto $\boldsymbol{U}_{l}$. The level $l$ edge element approximation of (2.1) reads as follows: Find $\boldsymbol{u}_{l} \in \boldsymbol{U}_{l}$ such that

$$
\boldsymbol{A}_{l} \boldsymbol{u}_{l}=\boldsymbol{f}_{l}
$$

We now consider local Hiptmair smoothers which smooth with respect to both edge basis functions and the gradients of nodal basis functions. The local smoothers are generalized Jacobi or Gauss-Seidel iterations with respect to appropriate subspace decompositions (cf. [23,24]). For $1 \leq l \leq L$, denoting by $\boldsymbol{b}_{l}^{E}$ the edge basis function associated with $E \in \mathcal{E}_{l}$ and by $\varphi_{l}^{p}$ the nodal basis function with supporting point $\boldsymbol{p} \in \mathcal{N}_{l}$, we define the set of edges $\widetilde{\mathcal{E}}_{l}$ and the set of vertices $\widetilde{\mathcal{N}}_{l}$ (see Fig. 1) on which the local smoothers are performed as follows:

$$
\begin{aligned}
& \widetilde{\mathcal{E}}_{l}=\left\{E \in \mathcal{E}_{l}: E \in \mathcal{E}_{l} \backslash \mathcal{E}_{l-1} \text { or } E \in \mathcal{E}_{l-1} \text { but } \operatorname{supp}\left(\boldsymbol{b}_{l}^{E}\right) \neq \operatorname{supp}\left(\boldsymbol{b}_{l-1}^{E}\right)\right\}, \\
& \widetilde{\mathcal{N}}_{l}=\left\{\boldsymbol{p} \in \mathcal{N}_{l}: \boldsymbol{p} \in \mathcal{N}_{l} \backslash \mathcal{N}_{l-1} \text { or } \boldsymbol{p} \in \mathcal{N}_{l-1} \text { but } \operatorname{supp}\left(\varphi_{l}^{\boldsymbol{p}}\right) \neq \operatorname{supp}\left(\varphi_{l-1}^{p}\right)\right\} .
\end{aligned}
$$


In fact, $\widetilde{\mathcal{E}}_{l}\left(\widetilde{\mathcal{N}}_{l}\right)$ is the set of new edges (vertices) and those local edges (vertices) where the support of the basis function has changed.

For the edge element space $\boldsymbol{U}_{L}$ we consider the following subspace decomposition ( $c f$. [23, 24]):

$$
\boldsymbol{U}_{L}=\boldsymbol{U}_{0}+\sum_{l=1}^{L}\left(\sum_{\boldsymbol{p} \in \tilde{\mathcal{N}}_{l}} \operatorname{Span}\left\{\nabla \varphi_{l}^{\boldsymbol{p}}\right\}+\sum_{E \in \widetilde{\mathcal{E}}_{l}} \operatorname{Span}\left\{\boldsymbol{b}_{l}^{E}\right\}\right)
$$

which follows from the discrete Helmholtz decomposition of the edge element space and the corresponding subspace decompositions (cf. [22]). For ease of notation, for $1 \leq l \leq L$ we write

$$
\bigcup_{i=1}^{N_{l}} \operatorname{Span}\left\{\nabla \varphi_{l}^{i}\right\}=\bigcup_{\boldsymbol{p} \in \widetilde{\mathcal{N}}_{l}} \operatorname{Span}\left\{\nabla \varphi_{l}^{p}\right\} \quad \text { and } \bigcup_{i=1}^{M_{l}} \operatorname{Span}\left\{\boldsymbol{b}_{l}^{i}\right\}=\bigcup_{E \in \widetilde{\mathcal{E}}_{l}} \operatorname{Span}\left\{\boldsymbol{b}_{l}^{E}\right\},
$$

where $N_{l}=\# \widetilde{\mathcal{N}}_{l}$ and $M_{l}=\# \widetilde{\mathcal{E}}_{l}$. We define the local subspace $\boldsymbol{U}_{l}^{i}$ according to

$$
\boldsymbol{U}_{l}^{i}= \begin{cases}\operatorname{Span}\left\{\nabla \varphi_{l}^{i}\right\}, & i=1, \ldots, N_{l}, \\ \operatorname{Span}\left\{\boldsymbol{b}_{l}^{j}\right\}, & i=N_{l}+j, j=1, \ldots, M_{l} .\end{cases}
$$

Let $\Omega_{l}^{i}$ be the support of the basis function spanning $\boldsymbol{U}_{l}^{i}$ and $h_{l, i}=\operatorname{diam}\left(\Omega_{l}^{i}\right)$. Then, for any $\boldsymbol{w} \in \boldsymbol{U}_{l}^{i}, i=$ $N_{l}+1, \ldots, N_{l}+M_{l}$, there holds (cf. [19], Lem. 3.1)

$$
\|\boldsymbol{w}\|_{0, \Omega} \leq C h_{l, i}\|\operatorname{curl} \boldsymbol{w}\|_{0, \Omega} .
$$

We further introduce the local projector $\boldsymbol{P}_{l}^{i}: \boldsymbol{U}_{L} \rightarrow \boldsymbol{U}_{l}^{i}$ according to

$$
\boldsymbol{a}\left(\boldsymbol{P}_{l}^{i} \boldsymbol{v}, \psi_{l}^{i}\right)=\boldsymbol{a}\left(\boldsymbol{v}, \psi_{l}^{i}\right), \quad \boldsymbol{v} \in \boldsymbol{U}_{L}, \psi_{l}^{i} \in \boldsymbol{U}_{l}^{i} .
$$

For $i=N_{l}+1, \ldots, N_{l}+M_{l},(2.7)$ and (2.8) imply that $\boldsymbol{P}_{l}^{i}$ is well defined for sufficiently small $h_{l, i}$ and satisfies (cf. [19], Prop. 3.1)

$$
\left\|\boldsymbol{P}_{l}^{i} \boldsymbol{v}\right\|_{\hat{\boldsymbol{a}}, \Omega_{l}^{i}} \leq C\|\boldsymbol{v}\|_{\hat{\boldsymbol{a}}, \Omega_{l}^{i}}, \quad \boldsymbol{v} \in \boldsymbol{U}_{L} .
$$

In particular, for $i=1, \ldots, N_{l}$ we have $\left\|\boldsymbol{P}_{l}^{i} \boldsymbol{v}\right\|_{0}^{2}=\left(v, \boldsymbol{P}_{l}^{i} \boldsymbol{v}\right)$ and hence, (2.9) also holds true. Moreover, for sufficiently small $h_{l, i}$ the following estimate is valid ( $c f$. [19], Lem. 4.4):

$$
\left(\boldsymbol{v}-\boldsymbol{P}_{l}^{i} \boldsymbol{v}, \boldsymbol{w}_{l}^{i}\right) \leq C h_{l, i}\left\|\boldsymbol{v}-\boldsymbol{P}_{l}^{i} \boldsymbol{v}\right\|_{0, \Omega_{l}^{i}}\left\|\operatorname{curl} \boldsymbol{w}_{l}^{i}\right\|_{0, \Omega_{l}^{i}}, \boldsymbol{v} \in \boldsymbol{U}_{L}, \boldsymbol{w}_{l}^{i} \in \boldsymbol{U}_{l}^{i}
$$

Obviously, (2.10) follows from (2.7) and the Cauchy-Schwarz inequality, if $i=N_{l}+1, \ldots, N_{l}+M_{l}$, whereas for $i=1, \ldots, N_{l}$ both sides of (2.10) are zero.

We define $\boldsymbol{A}_{l}^{i}: \boldsymbol{U}_{l}^{i} \rightarrow \boldsymbol{U}_{l}^{i}$ by

$$
\left(\boldsymbol{A}_{l}^{i} \boldsymbol{v}, \psi_{l}^{i}\right)=\boldsymbol{a}\left(\boldsymbol{v}, \psi_{l}^{i}\right), \quad \psi_{l}^{i}, \boldsymbol{v} \in \boldsymbol{U}_{l}^{i},
$$

and refer to $\boldsymbol{Q}_{l}^{i}: \boldsymbol{U}_{L} \rightarrow \boldsymbol{U}_{l}^{i}$ as the local $L^{2}$ projector. Clearly, (2.8) and (2.9) also imply the invertibility of $\boldsymbol{A}_{l}^{i}$. We note that $\hat{\boldsymbol{A}}_{l}, \hat{\boldsymbol{P}}_{l}, \hat{\boldsymbol{A}}_{l}^{i}$ and $\hat{\boldsymbol{P}}_{l}^{i}$ can be defined analogously by using $\hat{\boldsymbol{a}}(\cdot, \cdot)$ instead of $\boldsymbol{a}(\cdot, \cdot)$. For notational ease we set $\boldsymbol{U}_{0}:=\boldsymbol{U}_{0}^{N_{0}+M_{0}}$.

Let $\boldsymbol{R}_{l}^{J}: \boldsymbol{U}_{l} \rightarrow \boldsymbol{U}_{l}$ be the local Hiptmair-Jacobi smoother which performs Jacobi relaxations on the edges in $\widetilde{\mathcal{E}}_{l}$ and at the vertices in $\widetilde{\mathcal{N}}_{l}$, and let $\boldsymbol{R}_{l}^{G}: \boldsymbol{U}_{l} \rightarrow \boldsymbol{U}_{l}$ be the local Hiptmair-Gauss-Seidel smoother which 
performs Gauss-Seidel relaxations on the edges in $\widetilde{\mathcal{E}}_{l}$ and at the vertices in $\widetilde{\mathcal{N}}_{l}, 1 \leq l \leq L$. Moreover, we set $\boldsymbol{R}_{0}^{J}=\boldsymbol{R}_{0}^{G}=\boldsymbol{A}_{0}^{-1}$ on the initial mesh $\mathcal{T}_{0}$. Then $\boldsymbol{R}_{l}^{J}$ defines an additive smoother (cf. [9])

$$
\boldsymbol{R}_{l}^{J}:=\gamma \sum_{i=1}^{N_{l}+M_{l}}\left(\boldsymbol{A}_{l}^{i}\right)^{-1} \boldsymbol{Q}_{l}^{i}, \quad 1 \leq l \leq L,
$$

with a scaling factor $\gamma>0$, whereas $\boldsymbol{R}_{l}^{G}$ defines a multiplicative smoother

$$
\boldsymbol{R}_{l}^{G}:=\left(\boldsymbol{I}-\mathscr{E}_{l}\right) \boldsymbol{A}_{l}^{-1}, \quad \mathscr{E}_{l}:=\left(\boldsymbol{I}-\boldsymbol{P}_{l}^{N_{l}+M_{l}}\right) \cdots\left(\boldsymbol{I}-\boldsymbol{P}_{l}^{1}\right), \quad 1 \leq l \leq L,
$$

where $\boldsymbol{I}$ stands for the identity operator.

Remark 2.2. In case of nonsymmetric and indefinite linear second order elliptic boundary value problems, the associated bilinear forms become coercive on a subdomain for sufficiently small subdomain size and hence, the local symmetric and definite smoothers work well for LMM (cf. [13]). However, for the time-harmonic Maxwell problem the associated bilinear form is never positive on a subdomain. Therefore, in contrast to the algorithms presented in [14] where definite smoothers are used, we consider local smoothers based on the original indefinite problem ( $c f$. also Rem. 3.2 in [18]).

With $\boldsymbol{R}_{l}^{J}$ and $\boldsymbol{R}_{l}^{G}$ at hand, the LMM for the AEFEM approximation of (1.1a)-(1.1b) reads as follows:

Algorithm 2.3. Local Multigrid Methods (LMM).

Given an initial iterate $\boldsymbol{u}_{l}^{0} \in \boldsymbol{U}_{l}$, a sequence of approximations of the solution of (2.5) can be generated according to

$$
\boldsymbol{u}_{l}^{n+1}=\boldsymbol{u}_{l}^{n}+\boldsymbol{B}_{l}\left(\boldsymbol{f}_{l}-\boldsymbol{A}_{l} \boldsymbol{u}_{l}^{n}\right) .
$$

Here, for any $\boldsymbol{g} \in \boldsymbol{U}_{l}$ the multigrid operator $\boldsymbol{B}_{l}: \boldsymbol{U}_{l} \rightarrow \boldsymbol{U}_{l}: l \geq 0$ is recursively defined by means of:

$\boldsymbol{B}_{0}=\boldsymbol{A}_{0}^{-1}$ and $\boldsymbol{B}_{l} g=\boldsymbol{x}_{2}$, where

(i) Correction: $\boldsymbol{x}_{1}=\boldsymbol{B}_{l-1} \boldsymbol{Q}_{l-1} \boldsymbol{g}$;

(ii) Post-smoothing: $\boldsymbol{x}_{2}=\boldsymbol{x}_{1}+\boldsymbol{R}_{l}\left(\boldsymbol{g}-\boldsymbol{A}_{l} \boldsymbol{x}_{1}\right)$,

and the smoother can be either a local Hiptmair-Jacobi smoother $\boldsymbol{R}_{l}=\boldsymbol{R}_{l}^{J}$ or a local Hiptmair-Gauss-Seidel smoother $\boldsymbol{R}_{l}=\boldsymbol{R}_{l}^{G}$.

We point out that the local multigrid operator $\boldsymbol{B}_{l}$ can be treated as a preconditioner for GMRES applied to $(2.5)$ as it will be used in the numerical computations in Section 5.

\section{The ABstract SCHWARZ ThEORY}

In this section, we present an abstract framework for the convergence theory of LMM. The abstract theory depends on two important properties of the space decomposition of $\boldsymbol{U}_{L}$ which have been established in [24] (see also [23]) for $\boldsymbol{H}\left(\mathbf{c u r l} ; \Omega\right.$ )-elliptic problems, i.e., a stable multilevel decomposition of $\boldsymbol{U}_{L}$ and an associated global strengthened Cauchy-Schwarz inequality. We simply state the two properties as follows:

(S1) Stability of multilevel decomposition. For any function $\boldsymbol{v} \in \boldsymbol{U}_{L}$, there exists a decomposition

$$
\boldsymbol{v}=\boldsymbol{v}_{0}+\sum_{l=1}^{L} \sum_{i=1}^{N_{l}+M_{l}} \boldsymbol{v}_{l}^{i}, \quad \boldsymbol{v}_{0} \in \boldsymbol{U}_{0}, \boldsymbol{v}_{l}^{i} \in \boldsymbol{U}_{l}^{i},
$$

and a positive constant $C_{\text {stab }}$, independent of mesh sizes and mesh levels, such that

$$
\left\|\boldsymbol{v}_{0}\right\|_{\hat{\boldsymbol{a}}}^{2}+\sum_{l=1}^{L} \sum_{i=1}^{N_{l}+M_{l}}\left\|\boldsymbol{v}_{l}^{i}\right\|_{\hat{\boldsymbol{a}}}^{2} \leq C_{\text {stab }}\|\boldsymbol{v}\|_{\hat{\boldsymbol{a}}}^{2} .
$$


(S2) Global strengthened Cauchy-Schwarz inequality. For any functions

$$
\boldsymbol{v}_{l}^{i}, \boldsymbol{w}_{l}^{i} \in \boldsymbol{U}_{l}^{i}, \quad 1 \leq i \leq N_{l}+M_{l}, 0 \leq l \leq L,
$$

there exists a positive constant $C_{\text {orth }}$, independent of mesh sizes and mesh levels, such that

$$
\sum_{l=0}^{L} \sum_{i=1}^{N_{l}+M_{l}} \sum_{k=0}^{l-1} \sum_{j=1}^{N_{k}+M_{k}} \hat{\boldsymbol{a}}\left(\boldsymbol{v}_{l}^{i}, \boldsymbol{w}_{k}^{j}\right) \leq C_{\text {orth }}\left(\sum_{l=0}^{L} \sum_{i=1}^{N_{l}+M_{l}}\left\|\boldsymbol{v}_{l}^{i}\right\|_{\hat{\boldsymbol{a}}}^{2}\right)^{\frac{1}{2}}\left(\sum_{l=0}^{L} \sum_{i=1}^{N_{l}+M_{l}}\left\|\boldsymbol{w}_{l}^{i}\right\|_{\hat{\boldsymbol{a}}}^{2}\right)^{\frac{1}{2}} .
$$

Set $\boldsymbol{T}_{l}=\boldsymbol{R}_{l} \boldsymbol{A}_{l} \boldsymbol{P}_{l}, l=0,1, \ldots, L$, and $\boldsymbol{T}=\sum_{l=0}^{L} \boldsymbol{T}_{l}$. The abstract theory provides an estimate for the error operator

$$
\boldsymbol{E}=\left(\boldsymbol{I}-\boldsymbol{T}_{L}\right) \cdots\left(\boldsymbol{I}-\boldsymbol{T}_{1}\right)\left(\boldsymbol{I}-\boldsymbol{T}_{0}\right)=\prod_{l=0}^{L}\left(\boldsymbol{I}-\boldsymbol{T}_{l}\right),
$$

which can be deduced from the following statements:

(A1) There exist constants $C_{0}$ and $C_{1}$ such that

$$
\hat{\boldsymbol{a}}(\boldsymbol{v}, \boldsymbol{v}) \leq C_{0} \hat{\boldsymbol{a}}(\boldsymbol{T} \boldsymbol{v}, \boldsymbol{v})+C_{1} h_{0}^{2 s} \hat{\boldsymbol{a}}(\boldsymbol{v}, \boldsymbol{v}), \quad \boldsymbol{v} \in \boldsymbol{U}_{L} .
$$

(A2) Global spectral estimate. There exist constants $\omega \in(0,2)$ and $C_{2}>0$ such that for any $\boldsymbol{v} \in \boldsymbol{U}_{L}$,

$$
\sum_{l=0}^{L} \hat{\boldsymbol{a}}\left(\boldsymbol{T}_{l} \boldsymbol{E}_{l-1} \boldsymbol{v}, \boldsymbol{T}_{l} \boldsymbol{E}_{l-1} \boldsymbol{v}\right) \leq \omega \sum_{l=0}^{L} \hat{\boldsymbol{a}}\left(\boldsymbol{T}_{l} \boldsymbol{E}_{l-1} \boldsymbol{v}, \boldsymbol{E}_{l-1} \boldsymbol{v}\right)+C_{2} h_{0}^{2 s} \hat{\boldsymbol{a}}(\boldsymbol{v}, \boldsymbol{v}),
$$

where $\boldsymbol{E}_{l}=\left(\boldsymbol{I}-\boldsymbol{T}_{l}\right) \cdots\left(\boldsymbol{I}-\boldsymbol{T}_{0}\right)$ and $\boldsymbol{E}_{-1}=\boldsymbol{I}$.

(A3) There exist positive constants $C_{3}, C_{4}$ and $C_{5}$ such that for any $\boldsymbol{v} \in \boldsymbol{U}_{L}$

$\sum_{l=0}^{L} \sum_{k=0}^{l-1} \hat{\boldsymbol{a}}\left(\boldsymbol{T}_{l} \boldsymbol{v}, \boldsymbol{T}_{k} \boldsymbol{E}_{k-1} \boldsymbol{v}\right) \leq C_{3}\left(\sum_{l=0}^{L} \hat{\boldsymbol{a}}\left(\boldsymbol{T}_{l} \boldsymbol{v}, \boldsymbol{v}\right)+C_{4} h_{0}^{2 s} \hat{\boldsymbol{a}}(\boldsymbol{v}, \boldsymbol{v})\right)^{\frac{1}{2}} \cdot\left(\sum_{l=0}^{L} \hat{\boldsymbol{a}}\left(\boldsymbol{T}_{l} \boldsymbol{E}_{l-1} \boldsymbol{v}, \boldsymbol{E}_{l-1} \boldsymbol{v}\right)+C_{5} h_{0}^{2 s} \hat{\boldsymbol{a}}(\boldsymbol{v}, \boldsymbol{v})\right)^{\frac{1}{2}}$.

(A4) There exist positive constants $C_{6}, C_{7}$ and $C_{8}$ such that for any $\boldsymbol{v} \in \boldsymbol{U}_{L}$

$$
\sum_{l=0}^{L} \hat{\boldsymbol{a}}\left(\boldsymbol{T}_{l} \boldsymbol{v}, \boldsymbol{E}_{l-1} \boldsymbol{v}\right) \leq C_{6}\left(\sum_{l=0}^{L} \hat{\boldsymbol{a}}\left(\boldsymbol{T}_{l} \boldsymbol{v}, \boldsymbol{v}\right)+C_{7} h_{0}^{2 s} \hat{\boldsymbol{a}}(\boldsymbol{v}, \boldsymbol{v})\right)^{\frac{1}{2}} \cdot\left(\sum_{l=0}^{L} \hat{\boldsymbol{a}}\left(\boldsymbol{T}_{l} \boldsymbol{E}_{l-1} \boldsymbol{v}, \boldsymbol{E}_{l-1} \boldsymbol{v}\right)+C_{8} h_{0}^{2 s} \hat{\boldsymbol{a}}(\boldsymbol{v}, \boldsymbol{v})\right)^{\frac{1}{2}} .
$$

Based on the properties (S1) and (S2), in the next section we will apply a perturbation analysis to verify (A1)-(A3) for LMM with additive and multiplicative local Hiptmair smoothers. We note that (A4) can be derived similarly, and we thus do not give details. Combining (A3), (A4) leads to

$$
\sum_{l=0}^{L} \hat{\boldsymbol{a}}\left(\boldsymbol{T}_{l} \boldsymbol{v}, \boldsymbol{v}\right) \leq C_{9} \sum_{l=0}^{L} \hat{\boldsymbol{a}}\left(\boldsymbol{T}_{l} \boldsymbol{E}_{l-1} \boldsymbol{v}, \boldsymbol{E}_{l-1} \boldsymbol{v}\right)+C_{10} h_{0}^{2 s} \hat{\boldsymbol{a}}(\boldsymbol{v}, \boldsymbol{v}) .
$$

The main result of this paper reads as follows:

Theorem 3.1. For sufficiently small $h_{0},(\mathbf{A} 1)-(\mathbf{A} 4)$ are satisfied and the norm of the error operator $\boldsymbol{E}$ can be bounded as follows (cf. [37]):

$$
\hat{\boldsymbol{a}}(\boldsymbol{E} \boldsymbol{v}, \boldsymbol{E} \boldsymbol{v}) \leq \delta \hat{\boldsymbol{a}}(\boldsymbol{v}, \boldsymbol{v}), \quad \boldsymbol{v} \in \boldsymbol{U}_{L},
$$

where $\delta=1+K_{0} h_{0}^{2 s}-1 / K_{1}$. The positive constants $K_{0}$ and $K_{1}$ only depend on the shape regularity of the meshes and the wave number $\kappa$.

The theorem shows uniform convergence of LMM for (1.1a)-(1.1b) provided that the coarsest mesh is sufficiently fine. Similar to the estimates in [13], we can deduce uniform convergence of GMRES preconditioned by LMM. 


\section{Application to LMM With LOCAl Hiptmair SMoothers}

In this section, we verify (A1)-(A3) for both the additive local Hiptmair-Jacobi smoother and the multiplicative local Hiptmair-Gauss-Seidel smoother by a perturbation analysis which has been originally developed in [11] within a multigrid analysis for nonsymmetric and indefinite elliptic problems and has been also used in $[13,19]$.

\subsection{Local Hiptmair-Jacobi smoother}

Due to the definition of the local Hiptmair-Jacobi smoother $\boldsymbol{R}_{l}^{J}, l \geq 1$ in (2.11) and $\boldsymbol{R}_{0}^{J}=\boldsymbol{A}_{0}^{-1}$, we have

$$
\boldsymbol{T}_{0}=\boldsymbol{P}_{0}, \quad \boldsymbol{T}_{l}=\boldsymbol{R}_{l}^{J} \boldsymbol{A}_{l} \boldsymbol{P}_{l}=\gamma \sum_{i=1}^{N_{l}+M_{l}} \boldsymbol{P}_{l}^{i}, \quad l=1, \ldots, L .
$$

For any $\boldsymbol{u}, \boldsymbol{v} \in \boldsymbol{U}_{L}$, let $\boldsymbol{N}(\boldsymbol{u}, \boldsymbol{v})=\boldsymbol{a}(\boldsymbol{u}, \boldsymbol{v})-\hat{\boldsymbol{a}}(\boldsymbol{u}, \boldsymbol{v})=-\left(\kappa^{2}+1\right)(\boldsymbol{u}, \boldsymbol{v})$. Note that $\hat{\boldsymbol{A}}_{l}, \hat{\boldsymbol{P}}_{l}, \hat{\boldsymbol{A}}_{l}^{i}, \hat{\boldsymbol{P}}_{l}^{i}$ are defined based on the bilinear form $\hat{\boldsymbol{a}}(\cdot, \cdot)$. By definition

$$
\hat{\boldsymbol{a}}\left(\boldsymbol{P}_{l} \boldsymbol{u}, \boldsymbol{v}\right)=\boldsymbol{a}\left(\boldsymbol{u}, \hat{\boldsymbol{P}}_{l} \boldsymbol{v}\right)-\boldsymbol{N}\left(\boldsymbol{P}_{l} \boldsymbol{u}, \hat{\boldsymbol{P}}_{l} \boldsymbol{v}\right)=\hat{\boldsymbol{a}}\left(\hat{\boldsymbol{P}}_{l} \boldsymbol{u}, \boldsymbol{v}\right)+\boldsymbol{N}\left(\left(\boldsymbol{I}-\boldsymbol{P}_{l}\right) \boldsymbol{u}, \hat{\boldsymbol{P}}_{l} \boldsymbol{v}\right),
$$

and hence,

$$
\hat{\boldsymbol{a}}\left(\left(\boldsymbol{P}_{l}-\hat{\boldsymbol{P}}_{l}\right) \boldsymbol{u}, \boldsymbol{v}\right)=\boldsymbol{N}\left(\left(\boldsymbol{I}-\boldsymbol{P}_{l}\right) \boldsymbol{u}, \hat{\boldsymbol{P}}_{l} \boldsymbol{v}\right), \quad l=0,1, \ldots, L .
$$

For the subspaces in the decomposition (2.6) spanned by the gradients of nodal basis functions, we have

$$
\boldsymbol{P}_{l}^{i}=\hat{\boldsymbol{P}}_{l}^{i}, \quad i=1, \ldots, N_{l}, l=1, \ldots, L .
$$

Similar to (4.2), for $l=1, \ldots, L$ we also obtain

$$
\hat{\boldsymbol{a}}\left(\left(\boldsymbol{P}_{l}^{i}-\hat{\boldsymbol{P}}_{l}^{i}\right) \boldsymbol{u}, \boldsymbol{v}\right)=\boldsymbol{N}\left(\left(\boldsymbol{I}-\boldsymbol{P}_{l}^{i}\right) \boldsymbol{u}, \hat{\boldsymbol{P}}_{l}^{i} \boldsymbol{v}\right), \quad i=N_{l}+1, \ldots, N_{l}+M_{l}
$$

\subsubsection{Verification of (A1)}

Applying the stability of the multilevel decomposition (S1) for $\boldsymbol{H}(\mathbf{c u r l} ; \Omega)$-elliptic problems and using similar arguments as in [39], Lemmas 4.2, 4.7, results in

$$
\hat{\boldsymbol{a}}(\boldsymbol{v}, \boldsymbol{v}) \leq C \hat{\boldsymbol{a}}(\hat{\boldsymbol{T}} \boldsymbol{v}, \boldsymbol{v}), \quad \boldsymbol{v} \in \boldsymbol{U}_{L},
$$

where $\hat{\boldsymbol{T}}=\sum_{l=0}^{L} \hat{\boldsymbol{T}}_{l}, \hat{\boldsymbol{T}}_{l}=\hat{\boldsymbol{R}}_{l} \hat{\boldsymbol{A}}_{l} \hat{\boldsymbol{P}}_{l}$, and $\hat{\boldsymbol{R}}_{l}$ is the associated local Hiptmair-Jacobi or local Hiptmair-GaussSeidel smoother.

Lemma 4.1. Let $\boldsymbol{R}_{l}$ be given by (2.11). Then (A1) holds true.

Proof. An application of (4.5) yields

$$
\hat{\boldsymbol{a}}(\boldsymbol{v}, \boldsymbol{v}) \leq C \hat{\boldsymbol{a}}(\hat{\boldsymbol{T}} \boldsymbol{v}, \boldsymbol{v})=C \hat{\boldsymbol{a}}(\boldsymbol{T} \boldsymbol{v}, \boldsymbol{v})+C \sum_{l=0}^{L} \hat{\boldsymbol{a}}\left(\left(\hat{\boldsymbol{T}}_{l}-\boldsymbol{T}_{l}\right) \boldsymbol{v}, \boldsymbol{v}\right) .
$$


Combining (4.2)-(4.4) with (2.4) and (2.10), we deduce that

$$
\begin{aligned}
\sum_{l=0}^{L} \hat{\boldsymbol{a}}\left(\left(\hat{\boldsymbol{T}}_{l}-\boldsymbol{T}_{l}\right) \boldsymbol{v}, \boldsymbol{v}\right) & =\hat{\boldsymbol{a}}\left(\left(\hat{\boldsymbol{P}}_{0}-\boldsymbol{P}_{0}\right) \boldsymbol{v}, \boldsymbol{v}\right)+\gamma \sum_{l=1}^{L} \sum_{i=1}^{N_{l}+M_{l}} \hat{\boldsymbol{a}}\left(\left(\hat{\boldsymbol{P}}_{l}^{i}-\boldsymbol{P}_{l}^{i}\right) \boldsymbol{v}, \boldsymbol{v}\right) \\
& =\left(\kappa^{2}+1\right)\left(\left(\left(\boldsymbol{I}-\boldsymbol{P}_{0}\right) \boldsymbol{v}, \hat{\boldsymbol{P}}_{0} \boldsymbol{v}\right)+\gamma \sum_{l=1}^{L} \sum_{i=N_{l}+1}^{N_{l}+M_{l}}\left(\left(\boldsymbol{I}-\boldsymbol{P}_{l}^{i}\right) \boldsymbol{v}, \hat{\boldsymbol{P}}_{l}^{i} \boldsymbol{v}\right)\right) \\
& \leq C h_{0}^{s}\left\|\left(\boldsymbol{I}-\boldsymbol{P}_{0}\right) \boldsymbol{v}\right\|_{\hat{\boldsymbol{a}}}\left\|\hat{\boldsymbol{P}}_{0} \boldsymbol{v}\right\|_{\hat{\boldsymbol{a}}}+C \gamma \sum_{l=1}^{L} \sum_{i=N_{l}+1}^{N_{l}+M_{l}} h_{l, i}\left\|\left(\boldsymbol{I}-\boldsymbol{P}_{l}^{i}\right) \boldsymbol{v}\right\|_{\hat{\boldsymbol{a}}, \Omega_{l}^{i}\left\|\hat{\boldsymbol{P}}_{l}^{i} \boldsymbol{v}\right\|_{\hat{\boldsymbol{a}}}} \\
& \leq C h_{0}^{s}\|\boldsymbol{v}\|_{\hat{\boldsymbol{a}}}\left\|\hat{\boldsymbol{P}}_{0} \boldsymbol{v}\right\|_{\hat{\boldsymbol{a}}}+C \gamma \sum_{l=1}^{L} \sum_{i=N_{l}+1}^{N_{l}+M_{l}} h_{l, i}\|\boldsymbol{v}\|_{\hat{\boldsymbol{a}}, \Omega_{l}^{i}}\left\|\hat{\boldsymbol{P}}_{l}^{i} \boldsymbol{v}\right\|_{\hat{\boldsymbol{a}}} .
\end{aligned}
$$

Applying (4.6), (4.7), the Cauchy-Schwarz inequality, and Young's inequality gives

$$
\hat{\boldsymbol{a}}(\hat{\boldsymbol{T}} \boldsymbol{v}, \boldsymbol{v}) \leq C \hat{\boldsymbol{a}}(\boldsymbol{T} \boldsymbol{v}, \boldsymbol{v})+C h_{0}^{2 s}\|\boldsymbol{v}\|_{\hat{\boldsymbol{a}}}^{2}+C \gamma \sum_{l=1}^{L} \sum_{i=N_{l}+1}^{N_{l}+M_{l}} h_{l, i}^{2}\|\boldsymbol{v}\|_{\hat{\boldsymbol{a}}, \Omega_{l}^{i}}^{2} .
$$

Let $\widehat{\mathcal{T}}_{m}^{\mathcal{E}}=\bigcup_{l=1}^{L}\left\{T \in \mathcal{T}_{l}\left(\Omega_{l}^{E}\right): E \in \widetilde{\mathcal{E}}_{l}, \mathcal{G}(T)=m\right\}$. In view of $(2.2)$ we have

$$
\begin{aligned}
\sum_{l=1}^{L} \sum_{i=N_{l}+1}^{N_{l}+M_{l}} h_{l, i}^{2}\|\boldsymbol{v}\|_{\hat{\boldsymbol{a}}, \Omega_{l}^{i}}^{2} & \leq C \sum_{l=1}^{L} \sum_{E \in \tilde{\mathcal{E}}_{l}} \sum_{m=0}^{\infty} \sum_{\substack{T \in \mathcal{T}_{l}\left(\Omega_{l}^{E}\right), \mathcal{G}(T)=m}} h_{0}^{2} \theta^{2 m}\|\boldsymbol{v}\|_{\hat{\boldsymbol{a}}, T}^{2} \\
& =C h_{0}^{2} \sum_{m=0}^{\infty} \theta^{2 m} \sum_{\substack{T \in \widehat{\mathcal{T}}_{m}^{\mathcal{E}} \\
l}} \sum_{l \in \sigma(m, T)}\|\boldsymbol{v}\|_{\hat{\boldsymbol{a}}, T}^{2},
\end{aligned}
$$

where $\sigma(m, T)=\left\{l: T \in \widehat{\mathcal{T}}_{m}^{\mathcal{E}}, T \in \mathcal{T}_{l}, 1 \leq l \leq L\right\}$. The shape regularity of the meshes implies $\# \sigma(m, T) \leq C$. Observing that the elements in $\widehat{\mathcal{T}}_{m}^{\mathcal{E}}$ are nonintersecting and that the union of these elements is also a subset of $\Omega$, it follows that

$$
\sum_{l=1}^{L} \sum_{i=N_{l}+1}^{N_{l}+M_{l}} h_{l, i}^{2}\|\boldsymbol{v}\|_{\hat{\boldsymbol{a}}, \Omega_{l}^{i}}^{2} \leq C h_{0}^{2} \sum_{m=0}^{\infty} \theta^{2 m}\|\boldsymbol{v}\|_{\hat{\boldsymbol{a}}}^{2} \leq C h_{0}^{2}\|\boldsymbol{v}\|_{\hat{\boldsymbol{a}}}^{2} .
$$

Combining (4.6), (4.8), (4.9), and the fact that $h_{0}^{2} \leq h_{0}^{2 s}$ concludes the proof.

\subsubsection{Verification of (A2)}

As a prerequisite to verify (A2) we provide the following key estimate.

Lemma 4.2. For any functions $\boldsymbol{v}_{l}^{i} \in \boldsymbol{U}_{l}^{i}, 1 \leq i \leq N_{l}+M_{l}, 1 \leq l \leq L$, there holds

$$
\sum_{l=1}^{L} \sum_{i=1}^{N_{l}+M_{l}} h_{l, i}^{2}\left\|\sum_{k=1}^{l-1} \sum_{j=1}^{N_{k}+M_{k}} \boldsymbol{v}_{k}^{j}\right\|_{\hat{\boldsymbol{a}}, \Omega_{l}^{i}}^{2} \leq C \sum_{k=1}^{L} \sum_{j=1}^{N_{k}+M_{k}} h_{k, j}^{2}\left\|\boldsymbol{v}_{k}^{j}\right\|_{\hat{\boldsymbol{a}}}^{2} .
$$

Proof. Let $N_{k}^{q}$ be the number of elements in $\mathcal{T}_{k} \backslash \mathcal{T}_{k-1}$ which share $\boldsymbol{q} \in \widetilde{\mathcal{N}}_{k}$ and let $M_{k}^{E}$ be the number of elements in $\mathcal{T}_{k} \backslash \mathcal{T}_{k-1}$ which share $E \in \widetilde{\mathcal{E}}_{k}$. Then, we have

$$
\sum_{k=1}^{l-1} \sum_{j=1}^{N_{k}+M_{k}} \boldsymbol{v}_{k}^{j}=\sum_{k=1}^{l-1} \sum_{m=0}^{\infty} \sum_{\substack{K \in \mathcal{T}_{k} \backslash \mathcal{T}_{k-1} \\ \mathcal{G}(K)=m}}\left(\sum_{\boldsymbol{q} \in \mathcal{N}(K)} \tilde{\boldsymbol{v}}_{k}^{\boldsymbol{q}}+\sum_{E \in \mathcal{E}(K)} \tilde{\boldsymbol{v}}_{k}^{E}\right),
$$


where $\mathcal{E}(T)$ and $\mathcal{N}(T)$ are the sets of edges and vertices in $T$, respectively, and

$$
\tilde{\boldsymbol{v}}_{k}^{\boldsymbol{q}}=\left\{\begin{array}{ll}
\boldsymbol{v}_{k}^{\boldsymbol{q}} / N_{k}^{\boldsymbol{q}}, & \text { if } \boldsymbol{q} \in \widetilde{\mathcal{N}}_{k}, \\
0, & \text { otherwise }
\end{array} \quad \tilde{\boldsymbol{v}}_{k}^{E}= \begin{cases}\boldsymbol{v}_{k}^{E} / M_{k}^{E}, & \text { if } E \in \widetilde{\mathcal{E}}_{k}, \\
0, & \text { otherwise }\end{cases}\right.
$$

For any $K \in \mathcal{T}_{k}$, let $D_{k}^{K}=\left\{K^{\prime} \in \mathcal{T}_{k}: \overline{K^{\prime}} \cap \bar{K} \neq \emptyset\right\}$. For an element $T \in \mathcal{T}_{l}\left(\Omega_{l}^{i}\right)$, we assume that $\mathcal{G}(T)=n$. On this tetrahedron $T$, we find

$$
\begin{aligned}
\left\|\sum_{k=1}^{l-1} \sum_{j=N_{k}+1}^{N_{k}+M_{k}} \boldsymbol{v}_{k}^{j}\right\|_{\hat{\boldsymbol{a}}, T}^{2} & =\left\|\sum_{k=1}^{l-1} \sum_{m=0}^{\infty} \sum_{\substack{K \in \mathcal{T}_{k} \backslash \mathcal{T}_{k-1} \\
\mathcal{G}(K)=m}} \sum_{E \in \mathcal{E}(K)} \tilde{\boldsymbol{v}}_{k}^{E}\right\|_{\hat{\boldsymbol{a}, T}}^{2} \\
& \leq C\left(\sum_{k=1}^{l-1} \sum_{m=0}^{\infty} \sum_{\substack{K \in \mathcal{T}_{k} \backslash \mathcal{T}_{k-1} \\
T \subset D_{k}^{K}, \mathcal{G}(K)=m}} \sum_{E \in \mathcal{E}(K)}\left(\left\|\operatorname{curl} \tilde{\boldsymbol{v}}_{k}^{E}\right\|_{0, K}+\left\|\tilde{\boldsymbol{v}}_{k}^{E}\right\|_{0, K}\right) \frac{|T|^{1 / 2}}{|K|^{1 / 2}}\right)^{2} .
\end{aligned}
$$

We note that

$$
\begin{aligned}
& \left(\sum_{k=1}^{l-1} \sum_{m=0}^{\infty} \sum_{\substack{K \in \mathcal{T}_{k} \backslash \mathcal{T}_{k-1} \\
T \subset D_{k}^{K}, \mathcal{G}(K)=m}} \sum_{E \in \mathcal{E}(K)}\left(\left\|\operatorname{curl} \tilde{\boldsymbol{v}}_{k}^{E}\right\|_{0, K}+\left\|\tilde{\boldsymbol{v}}_{k}^{E}\right\|_{0, K}\right) \frac{|T|^{1 / 2}}{|K|^{1 / 2}}\right)^{2} \\
& \leq C\left(\sum_{k=1}^{l-1} \sum_{m=0}^{\infty} \sum_{\substack{K \in \mathcal{T}_{k} \backslash \mathcal{T}_{k-1} \\
T \subset D_{k}^{K}, \mathcal{G}(K)=m}} \sum_{E \in \mathcal{E}(K)}\left(\left\|\operatorname{curl} \tilde{\boldsymbol{v}}_{k}^{E}\right\|_{0, K}^{2}+\left\|\tilde{\boldsymbol{v}}_{k}^{E}\right\|_{0, K}^{2}\right) \frac{|T|^{2 / 3}}{|K|^{2 / 3}}\right) \\
& \left(\sum_{k=1}^{l-1} \sum_{m=0}^{\infty} \sum_{\substack{K \in \mathcal{T}_{k} \backslash \mathcal{T}_{k-1} \\
T \subset D_{k}^{K}, \mathcal{G}(K)=m}} \sum_{E \in \mathcal{E}(K)} \frac{|T|^{1 / 3}}{|K|^{1 / 3}}\right) .
\end{aligned}
$$

Since $T \subset D_{k}^{K}$, we know that $\mathcal{G}(K)=m$ for any $K \in \mathcal{T}_{k} \backslash \mathcal{T}_{k-1}$, whence $m \leq n+s_{0}$, where the integer $s_{0}$ only depends on the shape regularity of the meshes. We set

$$
\eta(m, T):=\left\{K: K \in \mathcal{T}_{k} \backslash \mathcal{T}_{k-1}, \mathcal{G}(K)=m, T \subset D_{k}^{K}, 1 \leq k \leq L\right\} .
$$

The local overlapping of $\left\{D_{k}^{K}: K \in \mathcal{T}_{k}\right\}$ implies $\# \eta(m, T) \leq C$. It follows that

$$
\begin{aligned}
\sum_{k=1}^{l-1} \sum_{m=0}^{\infty} \sum_{\substack{K \in \mathcal{T}_{k} \backslash \mathcal{T}_{k-1} \\
T \subset D_{k}^{K}, \mathcal{G}(K)=m}} \sum_{E \in \mathcal{E}(K)} \frac{|T|^{1 / 3}}{|K|^{1 / 3}} & \leq C \sum_{k=1}^{l-1} \sum_{m=0}^{n+s_{0}} \sum_{\substack{K \in \mathcal{T}_{k} \backslash \mathcal{T}_{k-1} \\
T \subset D_{k}^{K}, \mathcal{G}(K)=m}} \theta^{n-m} \\
& \leq C \sum_{m=0}^{n+s_{0}} \sum_{K \in \eta(m, T)} \theta^{n-m} \leq C \sum_{m=0}^{n+s_{0}} \theta^{n-m} \leq C .
\end{aligned}
$$


Hence,

$$
\left\|\sum_{k=1}^{l-1} \sum_{j=N_{k}+1}^{N_{k}+M_{k}} \boldsymbol{v}_{k}^{j}\right\|_{\hat{\boldsymbol{a}}, T}^{2} \leq C \sum_{k=1}^{l-1} \sum_{m=0}^{\infty} \sum_{\substack{K \in \mathcal{T}_{k} \backslash \mathcal{T}_{k-1} \\ T \subset D_{k}^{K}, \mathcal{G}(K)=m}} \sum_{E \in \mathcal{E}(K)}\left\|\tilde{\boldsymbol{v}}_{k}^{E}\right\|_{\hat{\boldsymbol{a}}, K}^{2} \frac{|T|^{2 / 3}}{|K|^{2 / 3}}
$$

Similar arguments yield

$$
\left\|\sum_{k=1}^{l-1} \sum_{j=1}^{N_{k}} \boldsymbol{v}_{k}^{j}\right\|_{\hat{\boldsymbol{a}}, T}^{2} \leq C \sum_{k=1}^{l-1} \sum_{m=0}^{\infty} \sum_{\substack{K \in \mathcal{T}_{k} \backslash \mathcal{T}_{k-1} \\ T \subset D_{k}^{K}, \mathcal{G}(K)=m}} \sum_{\boldsymbol{q} \in \mathcal{N}(K)}\left\|\tilde{\boldsymbol{v}}_{k}^{\boldsymbol{q}}\right\|_{\hat{\boldsymbol{a}}, K}^{2} \frac{|T|^{2 / 3}}{|K|^{2 / 3}}
$$

An application of (4.11) and (4.12) shows

$$
\begin{aligned}
& \sum_{l=1}^{L} \sum_{i=N_{l}+1}^{N_{l}+M_{l}} h_{l, i}^{2}\left\|\sum_{k=1}^{l-1} \sum_{j=1}^{N_{k}+M_{k}} \boldsymbol{v}_{k}^{j}\right\|_{\hat{\boldsymbol{a}}, \Omega_{l}^{i}}^{2} \leq C \sum_{l=1}^{L} \sum_{n=0}^{\infty} \sum_{E \in \tilde{\mathcal{E}}_{l}} \sum_{\substack{T \in \mathcal{T}_{l}\left(\Omega_{l}^{E}\right) \\
\mathcal{G}(T)=n}} h_{T}^{2} \\
& \cdot\left(\sum_{k=1}^{l-1} \sum_{m=0}^{\infty} \sum_{\substack{K \in \mathcal{T}_{k} \backslash \mathcal{T}_{k-1} \\
T \subset D_{k}^{K}, \mathcal{G}(K)=m}}\left(\sum_{E \in \mathcal{E}(K)}\left\|\tilde{\boldsymbol{v}}_{k}^{E}\right\|_{\hat{\boldsymbol{a}}, K}^{2}+\sum_{\boldsymbol{q} \in \mathcal{N}(K)}\left\|\tilde{\boldsymbol{v}}_{k}^{\boldsymbol{q}}\right\|_{\hat{\boldsymbol{a}}, K}^{2}\right) \frac{|T|^{2 / 3}}{|K|^{2 / 3}}\right) \\
& \leq C \sum_{k=1}^{L-1} \sum_{m=0}^{\infty} \sum_{\substack{K \in \mathcal{T}_{k} \backslash \mathcal{T}_{k-1} \\
\mathcal{G}(K)=m}}|K|^{2 / 3}\left(\sum_{E \in \mathcal{E}(K)}\left\|\tilde{\boldsymbol{v}}_{k}^{E}\right\|_{\hat{\boldsymbol{a}}, K}^{2}+\sum_{\boldsymbol{q} \in \mathcal{N}(K)}\left\|\tilde{\boldsymbol{v}}_{k}^{\boldsymbol{q}}\right\|_{\hat{\boldsymbol{a}}, K}^{2}\right) \\
& \cdot\left(\sum_{l=k+1}^{L} \sum_{n=0}^{\infty} \sum_{E \in \widetilde{\mathcal{E}}_{l}} \sum_{\substack{T \in \mathcal{T}_{l}\left(\Omega_{l}^{E}\right) \\
T \subset D_{k}^{K}, \mathcal{G}(T)=n}} \frac{|T|^{4 / 3}}{|K|^{4 / 3}}\right) .
\end{aligned}
$$

Let

$$
\tilde{\eta}(n, K, k)=\left\{T: T \in \mathcal{T}_{l}\left(\Omega_{l}^{E}\right), T \subset D_{k}^{K}, \mathcal{G}(T)=n, E \in \widetilde{\mathcal{E}}_{l}, 1 \leq k<l \leq L\right\} .
$$

Since $\mathcal{G}(K)=m$ for any $T \in \tilde{\eta}(n, K, k), T \subset D_{k}^{K}$, we have $m-s_{0} \leq n$, and the cardinality of the set $\tilde{\eta}(n, K, k)$ can be bounded as follows:

$$
\# \tilde{\eta}(n, K, k) \leq C \frac{|K|}{|T|} \leq C \theta^{3(m-n)}
$$

Furthermore, from (4.14) we deduce

$$
\sum_{l=k+1}^{L} \sum_{n=0}^{\infty} \sum_{E \in \widetilde{\mathcal{E}}_{l}} \sum_{T \in \mathcal{T}_{l}\left(\Omega_{l}^{E}\right)} \frac{|T|^{4 / 3}}{|K|^{4 / 3}} \leq C \sum_{n=0}^{\infty} \sum_{T \in \tilde{\eta}(n, K, k)} \frac{|T|^{4 / 3}}{|K|^{4 / 3}} \leq C \sum_{n=m-s_{0}}^{\infty} \theta^{n-m} \leq C .
$$


Combining (4.13) and (4.15) gives

$$
\begin{aligned}
\sum_{l=1}^{L} \sum_{i=N_{l}+1}^{N_{l}+M_{l}} h_{l, i}^{2}\left\|\sum_{k=1}^{l-1} \sum_{j=1}^{N_{k}+M_{k}} \boldsymbol{v}_{k}^{j}\right\|_{\hat{\boldsymbol{a}}, \Omega_{l}^{i}}^{2} & \leq C \sum_{k=1}^{L-1} \sum_{m=0}^{\infty} \sum_{\substack{K \in \mathcal{T}_{k} \backslash \mathcal{T}_{k-1} \\
\mathcal{G}(K)=m}}|K|^{2 / 3}\left(\sum_{E \in \mathcal{E}(K)}\left\|\tilde{\boldsymbol{v}}_{k}^{E}\right\|_{\hat{\boldsymbol{a}}, K}^{2}+\sum_{\boldsymbol{q} \in \mathcal{N}(K)}\left\|\tilde{\boldsymbol{v}}_{k}^{\boldsymbol{q}}\right\|_{\hat{\boldsymbol{a}}, K}^{2}\right) \\
& \leq C \sum_{k=1}^{L} \sum_{j=1}^{N_{k}+M_{k}} h_{k, j}^{2}\left\|\boldsymbol{v}_{k}^{j}\right\|_{\hat{\boldsymbol{a}}}^{2} .
\end{aligned}
$$

By similar arguments, we find

$$
\sum_{l=1}^{L} \sum_{i=1}^{N_{l}} h_{l, i}^{2}\left\|\sum_{k=1}^{l-1} \sum_{j=1}^{N_{k}+M_{k}} \boldsymbol{v}_{k}^{j}\right\|_{\hat{\boldsymbol{a}}, \Omega_{l}^{i}}^{2} \leq C \sum_{k=1}^{L} \sum_{j=1}^{N_{k}+M_{k}} h_{k, j}^{2}\left\|\boldsymbol{v}_{k}^{j}\right\|_{\hat{\boldsymbol{a}}}^{2} .
$$

Summing up (4.16) and (4.17) completes the proof.

The above lemma allows to verify (A2).

Lemma 4.3. Let $\boldsymbol{R}_{l}$ be given by (2.11). For sufficiently small initial mesh size $h_{0}$ and scaling parameter $\gamma$, there exists a constant $\omega \in(0,2)$ such that (A2) is satisfied.

Proof. In view of (2.4) and (4.2), it is obvious that

$$
\begin{aligned}
\hat{\boldsymbol{a}}\left(\boldsymbol{P}_{0} \boldsymbol{v}, \boldsymbol{P}_{0} \boldsymbol{v}\right) & =\hat{\boldsymbol{a}}\left(\boldsymbol{P}_{0} \boldsymbol{v}, \boldsymbol{v}\right)-\left(\kappa^{2}+1\right)\left(\left(\boldsymbol{I}-\boldsymbol{P}_{0}\right) \boldsymbol{v}, \hat{\boldsymbol{P}}_{0} \boldsymbol{P}_{0} \boldsymbol{v}\right) \\
& \leq \hat{\boldsymbol{a}}\left(\boldsymbol{P}_{0} \boldsymbol{v}, \boldsymbol{v}\right)+C h_{0}^{s}\left\|\left(\boldsymbol{I}-\boldsymbol{P}_{0}\right) \boldsymbol{v}\right\|_{\hat{\boldsymbol{a}}}\left\|\boldsymbol{P}_{0} \boldsymbol{v}\right\|_{\hat{\boldsymbol{a}}} \\
& \leq \hat{\boldsymbol{a}}\left(\boldsymbol{P}_{0} \boldsymbol{v}, \boldsymbol{v}\right)+\frac{1}{4}\left\|\boldsymbol{P}_{0} \boldsymbol{v}\right\|_{\hat{\boldsymbol{a}}}^{2}+C h_{0}^{2 s}\|\boldsymbol{v}\|_{\hat{\boldsymbol{a}}}^{2}
\end{aligned}
$$

which yields

$$
\hat{\boldsymbol{a}}\left(\boldsymbol{P}_{0} \boldsymbol{v}, \boldsymbol{P}_{0} \boldsymbol{v}\right) \leq \frac{4}{3} \hat{\boldsymbol{a}}\left(\boldsymbol{P}_{0} \boldsymbol{v}, \boldsymbol{v}\right)+C h_{0}^{2 s}\|\boldsymbol{v}\|_{\hat{\boldsymbol{a}}}^{2} .
$$

The local overlapping of $\left\{\Omega_{l}^{i}: 1 \leq i \leq N_{l}+M_{l}\right\}$ on each level implies

$$
\sum_{l=1}^{L} \hat{\boldsymbol{a}}\left(\boldsymbol{T}_{l} \boldsymbol{E}_{l-1} \boldsymbol{v}, \boldsymbol{T}_{l} \boldsymbol{E}_{l-1} \boldsymbol{v}\right) \leq C \gamma^{2} \sum_{l=1}^{L} \sum_{i=1}^{N_{l}+M_{l}} \hat{\boldsymbol{a}}\left(\boldsymbol{P}_{l}^{i} \boldsymbol{E}_{l-1} \boldsymbol{v}, \boldsymbol{P}_{l}^{i} \boldsymbol{E}_{l-1} \boldsymbol{v}\right)
$$

By definition of $\boldsymbol{P}_{l}^{i}, \hat{\boldsymbol{P}}_{l}^{i}$, and taking (4.3), (4.4) into account, we have

$$
\begin{aligned}
\sum_{l=1}^{L} \sum_{i=1}^{N_{l}+M_{l}} \hat{\boldsymbol{a}}\left(\boldsymbol{P}_{l}^{i} \boldsymbol{E}_{l-1} \boldsymbol{v}, \boldsymbol{P}_{l}^{i} \boldsymbol{E}_{l-1} \boldsymbol{v}\right)= & \sum_{l=1}^{L} \sum_{i=1}^{N_{l}+M_{l}} \hat{\boldsymbol{a}}\left(\boldsymbol{P}_{l}^{i} \boldsymbol{E}_{l-1} \boldsymbol{v}, \boldsymbol{E}_{l-1} \boldsymbol{v}\right) \\
& -\left(\kappa^{2}+1\right) \sum_{l=1}^{L} \sum_{i=N_{l}+1}^{N_{l}+M_{l}}\left(\left(\boldsymbol{I}-\boldsymbol{P}_{l}^{i}\right) \boldsymbol{E}_{l-1} \boldsymbol{v}, \hat{\boldsymbol{P}}_{l}^{i} \boldsymbol{P}_{l}^{i} \boldsymbol{E}_{l-1} \boldsymbol{v}\right) .
\end{aligned}
$$

Note that

$$
\begin{aligned}
\left(\kappa^{2}+1\right) \sum_{l=1}^{L} \sum_{i=N_{l}+1}^{N_{l}+M_{l}}\left(\left(\boldsymbol{I}-\boldsymbol{P}_{l}^{i}\right) \boldsymbol{E}_{l-1} \boldsymbol{v}, \hat{\boldsymbol{P}}_{l}^{i} \boldsymbol{P}_{l}^{i} \boldsymbol{E}_{l-1} \boldsymbol{v}\right) & \leq C \sum_{l=1}^{L} \sum_{i=N_{l}+1}^{N_{l}+M_{l}} h_{l, i}\left\|\boldsymbol{E}_{l-1} \boldsymbol{v}\right\|_{\hat{\boldsymbol{a}}, \Omega_{l}^{i}}\left\|\boldsymbol{P}_{l}^{i} \boldsymbol{E}_{l-1} \boldsymbol{v}\right\|_{\hat{\boldsymbol{a}}} \\
& \leq C \sum_{l=1}^{L} \sum_{i=N_{l}+1}^{N_{l}+M_{l}} h_{l, i}^{2}\left\|\boldsymbol{E}_{l-1} \boldsymbol{v}\right\|_{\hat{\boldsymbol{a}}, \Omega_{l}^{i}}^{2}+\frac{1}{2} \sum_{l=1}^{L} \sum_{i=N_{l}+1}^{N_{l}+M_{l}}\left\|\boldsymbol{P}_{l}^{i} \boldsymbol{E}_{l-1} \boldsymbol{v}\right\|_{\hat{\boldsymbol{a}}}^{2} .
\end{aligned}
$$


Hence,

$$
\sum_{l=1}^{L} \sum_{i=1}^{N_{l}+M_{l}} \hat{\boldsymbol{a}}\left(\boldsymbol{P}_{l}^{i} \boldsymbol{E}_{l-1} \boldsymbol{v}, \boldsymbol{P}_{l}^{i} \boldsymbol{E}_{l-1} \boldsymbol{v}\right) \leq C \sum_{l=1}^{L} \sum_{i=1}^{N_{l}+M_{l}} \hat{\boldsymbol{a}}\left(\boldsymbol{P}_{l}^{i} \boldsymbol{E}_{l-1} \boldsymbol{v}, \boldsymbol{E}_{l-1} \boldsymbol{v}\right)+C \sum_{l=1}^{L} \sum_{i=N_{l}+1}^{N_{l}+M_{l}} h_{l, i}^{2}\left\|\boldsymbol{E}_{l-1} \boldsymbol{v}\right\|_{\hat{\boldsymbol{a}}, \Omega_{l}^{i}}^{2} .
$$

Obviously, we have

$$
\boldsymbol{I}-\boldsymbol{E}_{l-1}=\sum_{k=1}^{l-1} \boldsymbol{T}_{k} \boldsymbol{E}_{k-1}+\boldsymbol{P}_{0}=\gamma \sum_{k=1}^{l-1} \sum_{j=1}^{N_{k}+M_{k}} \boldsymbol{P}_{k}^{j} \boldsymbol{E}_{k-1}+\boldsymbol{P}_{0}
$$

An application of (4.21), Lemma 4.2, the Cauchy-Schwarz inequality, and (4.9) gives

$$
\begin{aligned}
\sum_{l=1}^{L} \sum_{i=N_{l}+1}^{N_{l}+M_{l}} h_{l, i}^{2}\left\|\boldsymbol{E}_{l-1} \boldsymbol{v}\right\|_{\hat{\boldsymbol{a}}, \Omega_{l}^{i}}^{2} \leq & 2 \sum_{l=1}^{L} \sum_{i=N_{l}+1}^{N_{l}+M_{l}} h_{l, i}^{2}\|\boldsymbol{v}\|_{\hat{\boldsymbol{a}}, \Omega_{l}^{i}}^{2} \\
& +4 \gamma^{2} \sum_{l=1}^{L} \sum_{i=N_{l}+1}^{N_{l}+M_{l}} h_{l, i}^{2}\left\|\sum_{k=1}^{l-1} \sum_{j=1}^{N_{k}+M_{k}} \boldsymbol{P}_{k}^{j} \boldsymbol{E}_{k-1} \boldsymbol{v}\right\|_{\hat{\boldsymbol{a}}, \Omega_{l}^{i}}^{2}+4 \sum_{l=1}^{L} \sum_{i=N_{l}+1}^{N_{l}+M_{l}} h_{l, i}^{2}\left\|\boldsymbol{P}_{0} \boldsymbol{v}\right\|_{\hat{\boldsymbol{a}}, \Omega_{l}^{i}}^{2} \\
\leq & C h_{0}^{2}\|\boldsymbol{v}\|_{\hat{\boldsymbol{a}}}^{2}+C \sum_{l=1}^{L} \sum_{i=1}^{N_{l}+M_{l}} h_{l, i}^{2}\left\|\boldsymbol{P}_{l}^{i} \boldsymbol{E}_{l-1} \boldsymbol{v}\right\|_{\hat{\boldsymbol{a}}}^{2}+C h_{0}^{2}\left\|\boldsymbol{P}_{0} \boldsymbol{v}\right\|_{\hat{\boldsymbol{a}}}^{2} .
\end{aligned}
$$

Combining (4.20) and (4.22), for sufficiently small $h_{0}$ we have

$$
\begin{aligned}
\sum_{l=1}^{L} \sum_{i=1}^{N_{l}+M_{l}} \hat{\boldsymbol{a}}\left(\boldsymbol{P}_{l}^{i} \boldsymbol{E}_{l-1} \boldsymbol{v}, \boldsymbol{P}_{l}^{i} \boldsymbol{E}_{l-1} \boldsymbol{v}\right) \leq & C \sum_{l=1}^{L} \sum_{i=1}^{N_{l}+M_{l}} \hat{\boldsymbol{a}}\left(\boldsymbol{P}_{l}^{i} \boldsymbol{E}_{l-1} \boldsymbol{v}, \boldsymbol{E}_{l-1} \boldsymbol{v}\right) \\
& +C h_{0}^{2}\|\boldsymbol{v}\|_{\hat{\boldsymbol{a}}}^{2}+C h_{0}^{2}\left(\frac{4}{3} \hat{\boldsymbol{a}}\left(\boldsymbol{P}_{0} \boldsymbol{v}, \boldsymbol{v}\right)+C h_{0}^{2 s}\|\boldsymbol{v}\|_{\hat{\boldsymbol{a}}}^{2}\right) .
\end{aligned}
$$

Now, in view of (4.18), (4.19), and (4.23), it follows that

$$
\begin{aligned}
\sum_{l=0}^{L} \hat{\boldsymbol{a}}\left(\boldsymbol{T}_{l} \boldsymbol{E}_{l-1} \boldsymbol{v}, \boldsymbol{T}_{l} \boldsymbol{E}_{l-1} \boldsymbol{v}\right) \leq & C \gamma^{2} \sum_{l=1}^{L} \sum_{i=1}^{N_{l}+M_{l}} \hat{\boldsymbol{a}}\left(\boldsymbol{P}_{l}^{i} \boldsymbol{E}_{l-1} \boldsymbol{v}, \boldsymbol{E}_{l-1} \boldsymbol{v}\right) \\
& +\frac{4}{3}\left(1+C \gamma^{2} h_{0}^{2}\right) \hat{\boldsymbol{a}}\left(\boldsymbol{P}_{0} \boldsymbol{v}, \boldsymbol{v}\right)+C\left(\gamma^{2} h_{0}^{2}\left(1+h_{0}^{2 s}\right)+h_{0}^{2 s}\right) \hat{\boldsymbol{a}}(\boldsymbol{v}, \boldsymbol{v}) .
\end{aligned}
$$

We set $\omega:=\max \left\{C \gamma, \frac{4}{3}\left(1+C \gamma^{2} h_{0}^{2}\right)\right\}$. Then, (A2) follows by choosing $h_{0}$ and $\gamma$ sufficiently small such that $\omega \in(0,2)$.

\subsubsection{Verification of (A3)}

Based on the global strengthened Cauchy-Schwarz inequality (3.3) and (A2), we now verify (A3).

Lemma 4.4. Let $\boldsymbol{R}_{l}$ be given by (2.11). For sufficiently small $h_{0}$, there exist positive constants $C_{3}, C_{4}$ and $C_{5}$, which only depend on the shape regularity of the meshes, the wave number $\kappa$, the scaling parameter $\gamma$, and the initial mesh size $h_{0}$, such that (A3) is satisfied.

Proof. We first note that the global strengthened Cauchy-Schwarz inequality (3.3) directly gives rise to

$$
\begin{aligned}
\sum_{l=0}^{L} \sum_{k=0}^{l-1} \hat{\boldsymbol{a}}\left(\boldsymbol{T}_{l} \boldsymbol{v}, \boldsymbol{T}_{k} \boldsymbol{E}_{k-1} \boldsymbol{v}\right) \leq & C\left(\gamma^{2} \sum_{l=1}^{L} \sum_{i=1}^{N_{l}+M_{l}} \hat{\boldsymbol{a}}\left(\boldsymbol{P}_{l}^{i} \boldsymbol{v}, \boldsymbol{P}_{l}^{i} \boldsymbol{v}\right)+\hat{\boldsymbol{a}}\left(\boldsymbol{P}_{0} \boldsymbol{v}, \boldsymbol{P}_{0} \boldsymbol{v}\right)\right)^{\frac{1}{2}} \\
& \cdot\left(\gamma^{2} \sum_{l=1}^{L} \sum_{i=1}^{N_{l}+M_{l}} \hat{\boldsymbol{a}}\left(\boldsymbol{P}_{l}^{i} \boldsymbol{E}_{l-1} \boldsymbol{v}, \boldsymbol{P}_{l}^{i} \boldsymbol{E}_{l-1} \boldsymbol{v}\right)+\hat{\boldsymbol{a}}\left(\boldsymbol{P}_{0} \boldsymbol{v}, \boldsymbol{P}_{0} \boldsymbol{v}\right)\right)^{\frac{1}{2}} .
\end{aligned}
$$


By definition of $\boldsymbol{P}_{l}^{i}$ and $\hat{\boldsymbol{P}}_{l}^{i},(2.10)$, and (4.3), (4.4), similar arguments as in (4.18) show

$$
\begin{aligned}
\sum_{l=1}^{L} \sum_{i=1}^{N_{l}+M_{l}} \hat{\boldsymbol{a}}\left(\boldsymbol{P}_{l}^{i} \boldsymbol{v}, \boldsymbol{P}_{l}^{i} \boldsymbol{v}\right) & =\sum_{l=1}^{L} \sum_{i=1}^{N_{l}+M_{l}} \hat{\boldsymbol{a}}\left(\boldsymbol{P}_{l}^{i} \boldsymbol{v}, \boldsymbol{v}\right)-\sum_{l=1}^{L} \sum_{i=N_{l}+1}^{N_{l}+M_{l}}\left(\kappa^{2}+1\right)\left(\left(\boldsymbol{I}-\boldsymbol{P}_{l}^{i}\right) \boldsymbol{v}, \hat{\boldsymbol{P}}_{l}^{i} \boldsymbol{P}_{l}^{i} \boldsymbol{v}\right) \\
& \leq \sum_{l=1}^{L} \sum_{i=1}^{N_{l}+M_{l}} \hat{\boldsymbol{a}}\left(\boldsymbol{P}_{l}^{i} \boldsymbol{v}, \boldsymbol{v}\right)+C \sum_{l=1}^{L} \sum_{i=N_{l}+1}^{N_{l}+M_{l}} h_{l, i}\|\boldsymbol{v}\|_{\hat{\boldsymbol{a}}, \Omega_{l}^{i}}\left\|\boldsymbol{P}_{l}^{i} \boldsymbol{v}\right\|_{\hat{\boldsymbol{a}}} .
\end{aligned}
$$

Applying the Cauchy-Schwarz inequality, Young's inequality, and (4.9) gives

$$
\begin{aligned}
\sum_{l=1}^{L} \sum_{i=1}^{N_{l}+M_{l}} \hat{\boldsymbol{a}}\left(\boldsymbol{P}_{l}^{i} \boldsymbol{v}, \boldsymbol{P}_{l}^{i} \boldsymbol{v}\right) & \leq C \sum_{l=1}^{L} \sum_{i=1}^{N_{l}+M_{l}} \hat{\boldsymbol{a}}\left(\boldsymbol{P}_{l}^{i} \boldsymbol{v}, \boldsymbol{v}\right)+C \sum_{l=1}^{L} \sum_{i=N_{l}+1}^{N_{l}+M_{l}} h_{l, i}^{2}\|\boldsymbol{v}\|_{\hat{\boldsymbol{a}}, \Omega_{l}^{i}}^{2} \\
& \leq C \sum_{l=1}^{L} \sum_{i=1}^{N_{l}+M_{l}} \hat{\boldsymbol{a}}\left(\boldsymbol{P}_{l}^{i} \boldsymbol{v}, \boldsymbol{v}\right)+C h_{0}^{2} \hat{\boldsymbol{a}}(\boldsymbol{v}, \boldsymbol{v}) .
\end{aligned}
$$

The assertion now follows from (4.18), (4.23) and (4.24).

\subsection{Local Hiptmair-Gauss-Seidel smoother}

In this subsection, we consider the convergence of Algorithm 2.3 with the local Hiptmair-Gauss-Seidel smoother $\boldsymbol{R}_{l}^{G}, l \geq 1$ as given by (2.12). Observing $\boldsymbol{R}_{0}^{G}=\boldsymbol{A}_{0}^{-1}$, we have

$$
\boldsymbol{T}_{0}=\boldsymbol{P}_{0}, \quad \boldsymbol{T}_{l}=\boldsymbol{R}_{l}^{G} \boldsymbol{A}_{l} \boldsymbol{P}_{l}=\left(\boldsymbol{I}-\mathscr{E}_{l}\right) \boldsymbol{P}_{l}=\boldsymbol{I}-\mathscr{E}_{l}, \quad l=1, \ldots, L .
$$

Setting

$$
\mathscr{E}_{l}^{0}=\boldsymbol{I} \text { and } \mathscr{E}_{l}^{i}=\left(\boldsymbol{I}-\boldsymbol{P}_{l}^{i}\right) \cdots\left(\boldsymbol{I}-\boldsymbol{P}_{l}^{1}\right), i=1, \ldots, N_{l}+M_{l}, l \geq 1,
$$

it follows that $\mathscr{E}_{l}=\mathscr{E}_{l} N_{l}+M_{l}$. Obviously, we have

$$
\mathscr{E}_{l}^{i-1}-\mathscr{E}_{l}^{i}=\boldsymbol{P}_{l}^{i} \mathscr{E}_{l}^{i-1} \text { and } \boldsymbol{I}-\mathscr{E}_{l}=\sum_{i=1}^{N_{l}+M_{l}} \boldsymbol{P}_{l}^{i} \mathscr{E}_{l}^{i-1}
$$

From the identities (4.3) and (4.4), we further deduce (cf. [13], Sect. 4.2)

$$
\hat{\boldsymbol{a}}\left(\mathscr{E}_{l}^{i-1} \boldsymbol{v}, \mathscr{E}_{l}^{i-1} \boldsymbol{v}\right)=\hat{\boldsymbol{a}}\left(\mathscr{E}_{l}^{i} \boldsymbol{v}, \mathscr{E}_{l}^{i} \boldsymbol{v}\right)+\hat{\boldsymbol{a}}\left(\boldsymbol{P}_{l}^{i} \mathscr{E}_{l}^{i-1} \boldsymbol{v}, \boldsymbol{P}_{l}^{i} \mathscr{E}_{l}^{i-1} \boldsymbol{v}\right), \quad i=1, \ldots, N_{l}, \boldsymbol{v} \in \boldsymbol{U}_{L}
$$

Moreover, for $i=N_{l}+1, \ldots, N_{l}+M_{l}$ and $\boldsymbol{v} \in \boldsymbol{U}_{L}$ there holds

$$
\hat{\boldsymbol{a}}\left(\mathscr{E}_{l}^{i-1} \boldsymbol{v}, \mathscr{E}_{l}^{i-1} \boldsymbol{v}\right)=\hat{\boldsymbol{a}}\left(\mathscr{E}_{l}^{i} \boldsymbol{v}, \mathscr{E}_{l}^{i} \boldsymbol{v}\right)+\hat{\boldsymbol{a}}\left(\boldsymbol{P}_{l}^{i} \mathscr{E}_{l}^{i-1} \boldsymbol{v}, \boldsymbol{P}_{l}^{i} \mathscr{E}_{l}^{i-1} \boldsymbol{v}\right)-2 \boldsymbol{N}\left(\left(\boldsymbol{I}-\boldsymbol{P}_{l}^{i}\right) \mathscr{E}_{l}^{i-1} \boldsymbol{v}, \hat{\boldsymbol{P}}_{l}^{i} \boldsymbol{P}_{l}^{i} \mathscr{E}_{l}^{i-1} \boldsymbol{v}\right)
$$

Summing the above two identities over all $i$ results in

$$
\sum_{i=1}^{N_{l}+M_{l}} \hat{\boldsymbol{a}}\left(\boldsymbol{P}_{l}^{i} \mathscr{E}_{l}^{i-1} \boldsymbol{v}, \boldsymbol{P}_{l}^{i} \mathscr{E}_{l}^{i-1} \boldsymbol{v}\right)=2 \sum_{i=N_{l}+1}^{N_{l}+M_{l}} \boldsymbol{N}\left(\left(\boldsymbol{I}-\boldsymbol{P}_{l}^{i}\right) \mathscr{E}_{l}^{i-1} \boldsymbol{v}, \hat{\boldsymbol{P}}_{l}^{i} \boldsymbol{P}_{l}^{i} \mathscr{E}_{l}^{i-1} \boldsymbol{v}\right)+2 \hat{\boldsymbol{a}}\left(\boldsymbol{T}_{l} \boldsymbol{v}, \boldsymbol{v}\right)-\hat{\boldsymbol{a}}\left(\boldsymbol{T}_{l} \boldsymbol{v}, \boldsymbol{T}_{l} \boldsymbol{v}\right)
$$

\subsubsection{Verification of (A1)}

We recall that in Lemma 4.1 we have obtained the estimate

$$
\hat{\boldsymbol{a}}(\boldsymbol{v}, \boldsymbol{v}) \leq C\left(\hat{\boldsymbol{a}}\left(\boldsymbol{P}_{0} \boldsymbol{v}, \boldsymbol{v}\right)+\sum_{l=1}^{L} \sum_{i=1}^{N_{l}+M_{l}} \hat{\boldsymbol{a}}\left(\boldsymbol{P}_{l}^{i} \boldsymbol{v}, \boldsymbol{v}\right)+h_{0}^{2 s} \hat{\boldsymbol{a}}(\boldsymbol{v}, \boldsymbol{v})\right), \quad \boldsymbol{v} \in \boldsymbol{U}_{L},
$$

which is a basic tool in the verification of (A1). 
Lemma 4.5. Let $\boldsymbol{R}_{l}$ be given by (2.12). For sufficiently small $h_{0}$, (A1) holds true.

Proof. Obviously, in view of (2.10), (4.3), (4.4), and (4.9) we have

$$
\begin{aligned}
\sum_{l=1}^{L} \sum_{i=1}^{N_{l}+M_{l}} \hat{\boldsymbol{a}}\left(\boldsymbol{P}_{l}^{i} \boldsymbol{v}, \boldsymbol{v}\right) & =\sum_{l=1}^{L} \sum_{i=1}^{N_{l}+M_{l}} \hat{\boldsymbol{a}}\left(\boldsymbol{P}_{l}^{i} \boldsymbol{v}, \boldsymbol{P}_{l}^{i} \boldsymbol{v}\right)-\sum_{l=1}^{L} \sum_{i=N_{l}+1}^{N_{l}+M_{l}} \boldsymbol{N}\left(\left(\boldsymbol{I}-\boldsymbol{P}_{l}^{i}\right) \boldsymbol{v}, \hat{\boldsymbol{P}}_{l}^{i} \boldsymbol{P}_{l}^{i} \boldsymbol{v}\right) \\
& \leq \sum_{l=1}^{L} \sum_{i=1}^{N_{l}+M_{l}} \hat{\boldsymbol{a}}\left(\boldsymbol{P}_{l}^{i} \boldsymbol{v}, \boldsymbol{P}_{l}^{i} \boldsymbol{v}\right)+C \sum_{l=1}^{L} \sum_{i=N_{l}+1}^{N_{l}+M_{l}} h_{l, i}\|\boldsymbol{v}\|_{\hat{\boldsymbol{a}}, \Omega_{l}^{i}}\left\|\boldsymbol{P}_{l}^{i} \boldsymbol{v}\right\|_{\hat{\boldsymbol{a}}} \\
& \leq C \sum_{l=1}^{L} \sum_{i=1}^{N_{l}+M_{l}} \hat{\boldsymbol{a}}\left(\boldsymbol{P}_{l}^{i} \boldsymbol{v}, \boldsymbol{P}_{l}^{i} \boldsymbol{v}\right)+C \sum_{l=1}^{L} \sum_{i=N_{l}+1}^{N_{l}+M_{l}} h_{l, i}^{2}\|\boldsymbol{v}\|_{\hat{\boldsymbol{a}}, \Omega_{l}^{i}}^{2} \\
& \leq C \sum_{l=1}^{L} \sum_{i=1}^{N_{l}+M_{l}} \hat{\boldsymbol{a}}\left(\boldsymbol{P}_{l}^{i} \boldsymbol{v}, \boldsymbol{P}_{l}^{i} \boldsymbol{v}\right)+C h_{0}^{2} \hat{\boldsymbol{a}}(\boldsymbol{v}, \boldsymbol{v}) .
\end{aligned}
$$

Taking

$$
\boldsymbol{I}-\mathscr{E}_{l}^{i-1}=\sum_{j=1}^{i-1} \boldsymbol{P}_{l}^{j} \mathscr{E}_{l}^{j-1}
$$

into account, it follows that

$$
\hat{\boldsymbol{a}}\left(\boldsymbol{P}_{l}^{i} \boldsymbol{v}, \boldsymbol{P}_{l}^{i} \boldsymbol{v}\right)=\hat{\boldsymbol{a}}\left(\boldsymbol{P}_{l}^{i} \boldsymbol{v}, \boldsymbol{P}_{l}^{i} \mathscr{E}_{l}^{i-1} \boldsymbol{v}\right)+\hat{\boldsymbol{a}}\left(\boldsymbol{P}_{l}^{i} \boldsymbol{v}, \sum_{j=1}^{i-1} \boldsymbol{P}_{l}^{i} \boldsymbol{P}_{l}^{j} \mathscr{E}_{l}^{j-1} \boldsymbol{v}\right)
$$

Due to the above identity, the local overlapping of $\left\{\Omega_{l}^{i}: 1 \leq i \leq N_{l}+M_{l}\right\}$ on each level implies (cf. [9], Lem. 5.1)

$$
\sum_{i=1}^{N_{l}+M_{l}} \hat{\boldsymbol{a}}\left(\boldsymbol{P}_{l}^{i} \boldsymbol{v}, \boldsymbol{P}_{l}^{i} \boldsymbol{v}\right) \leq C \sum_{i=1}^{N_{l}+M_{l}} \hat{\boldsymbol{a}}\left(\boldsymbol{P}_{l}^{i} \mathscr{E}_{l}^{i-1} \boldsymbol{v}, \boldsymbol{P}_{l}^{i} \mathscr{E}_{l}^{i-1} \boldsymbol{v}\right)
$$

Using (2.10) and (4.26), we get

$$
\sum_{i=1}^{N_{l}+M_{l}} \hat{\boldsymbol{a}}\left(\boldsymbol{P}_{l}^{i} \mathscr{E}_{l}^{i-1} \boldsymbol{v}, \boldsymbol{P}_{l}^{i} \mathscr{E}_{l}^{i-1} \boldsymbol{v}\right) \leq 2 \hat{\boldsymbol{a}}\left(\boldsymbol{T}_{l} \boldsymbol{v}, \boldsymbol{v}\right)-\hat{\boldsymbol{a}}\left(\boldsymbol{T}_{l} \boldsymbol{v}, \boldsymbol{T}_{l} \boldsymbol{v}\right)+C \sum_{i=N_{l}+1}^{N_{l}+M_{l}} h_{l, i}\left\|\mathscr{E}_{l}^{i-1} \boldsymbol{v}\right\|_{\hat{\boldsymbol{a}}, \Omega_{l}^{i}}\left\|\boldsymbol{P}_{l}^{i} \mathscr{E}_{l}^{i-1} \boldsymbol{v}\right\|_{\hat{\boldsymbol{a}}}
$$

Then, an application of Young's inequality results in

$$
\sum_{i=1}^{N_{l}+M_{l}} \hat{\boldsymbol{a}}\left(\boldsymbol{P}_{l}^{i} \mathscr{E}_{l}^{i-1} \boldsymbol{v}, \boldsymbol{P}_{l}^{i} \mathscr{E}_{l}^{i-1} \boldsymbol{v}\right) \leq C \hat{\boldsymbol{a}}\left(\boldsymbol{T}_{l} \boldsymbol{v}, \boldsymbol{v}\right)+C \sum_{i=N_{l}+1}^{N_{l}+M_{l}} h_{l, i}^{2}\left\|\mathscr{E}_{l}^{i-1} \boldsymbol{v}\right\|_{\hat{\boldsymbol{a}}, \Omega_{l}^{i}}^{2}
$$

In order to deal with the second term on the right-hand side of (4.31), the limited interaction property and (4.29) imply

$$
\begin{aligned}
\sum_{i=N_{l}+1}^{N_{l}+M_{l}} h_{l, i}^{2}\left\|\mathscr{E}_{l}^{i-1} \boldsymbol{v}\right\|_{\hat{\boldsymbol{a}}, \Omega_{l}^{i}}^{2} & \leq 2 \sum_{i=N_{l}+1}^{N_{l}+M_{l}} h_{l, i}^{2}\|\boldsymbol{v}\|_{\hat{\boldsymbol{a}}, \Omega_{l}^{i}}^{2}+2 \sum_{i=N_{l}+1}^{N_{l}+M_{l}} h_{l, i}^{2}\left\|\sum_{j=1}^{i-1} \boldsymbol{P}_{l}^{j} \mathscr{E}_{l}^{j-1} \boldsymbol{v}\right\|_{\hat{\boldsymbol{a}}, \Omega_{l}^{i}}^{2} \\
& \leq 2 \sum_{i=N_{l}+1}^{N_{l}+M_{l}} h_{l, i}^{2}\|\boldsymbol{v}\|_{\hat{\boldsymbol{a}}, \Omega_{l}^{i}}^{2}+C \sum_{j=1}^{N_{l}+M_{l}} \sum_{\substack{i=N_{l}+1 \\
\Omega_{l}^{i} \cap \Omega_{l}^{j} \neq \emptyset}}^{N_{l}+M_{l}} h_{l, i}^{2}\left\|\boldsymbol{P}_{l}^{j} \mathscr{E}_{l}^{j-1} \boldsymbol{v}\right\|_{\hat{\boldsymbol{a}}, \Omega_{l}^{i}}^{2} \\
& \leq 2 \sum_{i=N_{l}+1}^{N_{l}+M_{l}} h_{l, i}^{2}\|\boldsymbol{v}\|_{\hat{\boldsymbol{a}}, \Omega_{l}^{i}}^{2}+C \sum_{j=1}^{N_{l}+M_{l}} h_{l, j}^{2}\left\|\boldsymbol{P}_{l}^{j} \mathscr{E}_{l}^{j-1} \boldsymbol{v}\right\|_{\hat{\boldsymbol{a}}}^{2} .
\end{aligned}
$$


Obviously, for sufficiently small $h_{0},(4.31)$ and (4.32) give rise to

$$
\sum_{i=1}^{N_{l}+M_{l}} \hat{\boldsymbol{a}}\left(\boldsymbol{P}_{l}^{i} \mathscr{E}_{l}^{i-1} \boldsymbol{v}, \boldsymbol{P}_{l}^{i} \mathscr{E}_{l}^{i-1} \boldsymbol{v}\right) \leq C \hat{\boldsymbol{a}}\left(\boldsymbol{T}_{l} \boldsymbol{v}, \boldsymbol{v}\right)+C \sum_{i=N_{l}+1}^{N_{l}+M_{l}} h_{l, i}^{2}\|\boldsymbol{v}\|_{\hat{\boldsymbol{a}}, \Omega_{l}^{i}}^{2}
$$

Summing over $l$, it follows that

$$
\begin{aligned}
\sum_{l=1}^{L} \sum_{i=1}^{N_{l}+M_{l}} \hat{\boldsymbol{a}}\left(\boldsymbol{P}_{l}^{i} \mathscr{E}_{l}^{i-1} \boldsymbol{v}, \boldsymbol{P}_{l}^{i} \mathscr{E}_{l}^{i-1} \boldsymbol{v}\right) & \leq C \sum_{l=1}^{L} \hat{\boldsymbol{a}}\left(\boldsymbol{T}_{l} \boldsymbol{v}, \boldsymbol{v}\right)+C \sum_{l=1}^{L} \sum_{i=N_{l}+1}^{N_{l}+M_{l}} h_{l, i}^{2}\|\boldsymbol{v}\|_{\hat{\boldsymbol{a}}, \Omega_{l}^{i}}^{2} \\
& \leq C \sum_{l=1}^{L} \hat{\boldsymbol{a}}\left(\boldsymbol{T}_{l} \boldsymbol{v}, \boldsymbol{v}\right)+C h_{0}^{2} \hat{\boldsymbol{a}}(\boldsymbol{v}, \boldsymbol{v})
\end{aligned}
$$

which together with (4.27), (4.28) and (4.30) allows us to conclude the proof.

\subsubsection{Verification of (A2)}

For the verification of (A2), Lemma 4.2 also plays a key role in the analysis.

Lemma 4.6. Let $\boldsymbol{R}_{i}$ be given by (2.12). For sufficiently small $h_{0}$, there exists a constant $\omega \in(0,2)$ such that (A2) holds true.

Proof. In view of (4.18), it suffices to deal with the remaining terms on the left-hand side of (A2). By means of the limited interaction property on each level we have

$$
\sum_{l=1}^{L} \hat{\boldsymbol{a}}\left(\boldsymbol{T}_{l} \boldsymbol{E}_{l-1} \boldsymbol{v}, \boldsymbol{T}_{l} \boldsymbol{E}_{l-1} \boldsymbol{v}\right) \leq C \sum_{l=1}^{L} \sum_{i=1}^{N_{l}+M_{l}} \hat{\boldsymbol{a}}\left(\boldsymbol{P}_{l}^{i} \mathscr{E}_{l}^{i-1} \boldsymbol{E}_{l-1} \boldsymbol{v}, \boldsymbol{P}_{l}^{i} \mathscr{E}_{l}^{i-1} \boldsymbol{E}_{l-1} \boldsymbol{v}\right)
$$

Using (2.10) and (4.26) again, we find

$$
\begin{aligned}
\sum_{i=1}^{N_{l}+M_{l}} \hat{\boldsymbol{a}}\left(\boldsymbol{P}_{l}^{i} \mathscr{E}_{l}^{i-1} \boldsymbol{E}_{l-1} \boldsymbol{v}, \boldsymbol{P}_{l}^{i} \mathscr{E}_{l}^{i-1} \boldsymbol{E}_{l-1} \boldsymbol{v}\right) \leq & 2 \hat{\boldsymbol{a}}\left(\boldsymbol{T}_{l} \boldsymbol{E}_{l-1} \boldsymbol{v}, \boldsymbol{E}_{l-1} \boldsymbol{v}\right)-\hat{\boldsymbol{a}}\left(\boldsymbol{T}_{l} \boldsymbol{E}_{l-1} \boldsymbol{v}, \boldsymbol{T}_{l} \boldsymbol{E}_{l-1} \boldsymbol{v}\right) \\
& +C \sum_{i=N_{l}+1}^{N_{l}+M_{l}} h_{l, i}\left\|\mathscr{E}_{l}^{i-1} \boldsymbol{E}_{l-1} \boldsymbol{v}\right\|_{\hat{\boldsymbol{a}}, \Omega_{l}^{i}}\left\|\boldsymbol{P}_{l}^{i} \mathscr{E}_{l}^{i-1} \boldsymbol{E}_{l-1} \boldsymbol{v}\right\|_{\hat{\boldsymbol{a}}}
\end{aligned}
$$

Young's inequality and (4.32) imply

$$
\begin{aligned}
C \sum_{i=N_{l}+1}^{N_{l}+M_{l}} h_{l, i}\left\|\mathscr{E}_{l}^{i-1} \boldsymbol{E}_{l-1} \boldsymbol{v}\right\|_{\hat{\boldsymbol{a}}, \Omega_{l}^{i}}\left\|\boldsymbol{P}_{l}^{i} \mathscr{E}_{l}^{i-1} \boldsymbol{E}_{l-1} \boldsymbol{v}\right\|_{\hat{\boldsymbol{a}}} \leq & C \sum_{i=N_{l}+1}^{N_{l}+M_{l}} h_{l, i}^{2}\left\|\mathscr{E}_{l}^{i-1} \boldsymbol{E}_{l-1} \boldsymbol{v}\right\|_{\hat{\boldsymbol{a}}, \Omega_{l}^{i}}^{2}+\frac{1}{2} \sum_{i=N_{l}+1}^{N_{l}+M_{l}}\left\|\boldsymbol{P}_{l}^{i} \mathscr{E}_{l}^{i-1} \boldsymbol{E}_{l-1} \boldsymbol{v}\right\|_{\hat{\boldsymbol{a}}}^{2} \\
\leq & C \sum_{i=N_{l}+1}^{N_{l}+M_{l}} h_{l, i}^{2}\left\|\boldsymbol{E}_{l-1} \boldsymbol{v}\right\|_{\hat{\boldsymbol{a}}, \Omega_{l}^{i}}^{2}+C \sum_{i=1}^{N_{l}+M_{l}} h_{l, i}^{2}\left\|\boldsymbol{P}_{l}^{i} \mathscr{E}_{l}^{i-1} \boldsymbol{E}_{l-1} \boldsymbol{v}\right\|_{\hat{\boldsymbol{a}}}^{2} \\
& +\frac{1}{2} \sum_{i=N_{l}+1}^{N_{l}+M_{l}}\left\|\boldsymbol{P}_{l}^{i} \mathscr{E}_{l}^{i-1} \boldsymbol{E}_{l-1} \boldsymbol{v}\right\|_{\hat{\boldsymbol{a}}}^{2} .
\end{aligned}
$$

Consequently, in view of (4.35), (4.36), by summation over $l$ we find that for sufficiently small initial mesh size $h_{0}$ there holds

$$
\begin{aligned}
\sum_{l=1}^{L} \sum_{i=1}^{N_{l}+M_{l}} \hat{\boldsymbol{a}}\left(\boldsymbol{P}_{l}^{i} \mathscr{E}_{l}^{i-1} \boldsymbol{E}_{l-1} \boldsymbol{v}, \boldsymbol{P}_{l}^{i} \mathscr{E}_{l}{ }^{i-1} \boldsymbol{E}_{l-1} \boldsymbol{v}\right) \leq & 2 C \sum_{l=1}^{L} \hat{\boldsymbol{a}}\left(\boldsymbol{T}_{l} \boldsymbol{E}_{l-1} \boldsymbol{v}, \boldsymbol{E}_{l-1} \boldsymbol{v}\right)-C \sum_{l=1}^{L} \hat{\boldsymbol{a}}\left(\boldsymbol{T}_{l} \boldsymbol{E}_{l-1} \boldsymbol{v}, \boldsymbol{T}_{l} \boldsymbol{E}_{l-1} \boldsymbol{v}\right) \\
& +C_{11} \sum_{l=1}^{L} \sum_{i=N_{l}+1}^{N_{l}+M_{l}} h_{l, i}^{2}\left\|\boldsymbol{E}_{l-1} \boldsymbol{v}\right\|_{\hat{\boldsymbol{a}}, \Omega_{l}^{i}}^{2} .
\end{aligned}
$$


Observing

$$
\boldsymbol{I}-\boldsymbol{E}_{l-1}=\sum_{k=1}^{l-1} \boldsymbol{T}_{k} \boldsymbol{E}_{k-1}+\boldsymbol{P}_{0}=\sum_{k=1}^{l-1} \sum_{j=1}^{N_{k}+M_{k}} \boldsymbol{P}_{k}^{j} \mathscr{E}_{k}^{j-1} \boldsymbol{E}_{k-1}+\boldsymbol{P}_{0},
$$

by Lemma 4.2 and similar arguments as in (4.22) we obtain

$$
\begin{aligned}
\sum_{l=1}^{L} \sum_{i=N_{l}+1}^{N_{l}+M_{l}} h_{l, i}^{2}\left\|\boldsymbol{E}_{l-1} \boldsymbol{v}\right\|_{\hat{\boldsymbol{a}}, \Omega_{l}^{i}}^{2} \leq & 2 \sum_{l=1}^{L} \sum_{i=N_{l}+1}^{N_{l}+M_{l}} h_{l, i}^{2}\|\boldsymbol{v}\|_{\hat{\boldsymbol{a}}, \Omega_{l}^{i}}^{2} \\
& +2 \sum_{l=1}^{L} \sum_{i=N_{l}+1}^{N_{l}+M_{l}} h_{l, i}^{2}\left\|\sum_{k=1}^{l-1} \sum_{j=1}^{N_{k}+M_{k}} \boldsymbol{P}_{k}^{j} \mathscr{E}_{k}^{j-1} \boldsymbol{E}_{k-1} \boldsymbol{v}+\boldsymbol{P}_{0} \boldsymbol{v}\right\|_{\hat{a}, \Omega_{l}^{i}}^{2} \\
\leq & C h_{0}^{2} \hat{\boldsymbol{a}}(\boldsymbol{v}, \boldsymbol{v})+C h_{0}^{2} \hat{\boldsymbol{a}}\left(\boldsymbol{P}_{0} \boldsymbol{v}, \boldsymbol{P}_{0} \boldsymbol{v}\right)+C \sum_{l=1}^{L} \sum_{i=1}^{N_{l}+M_{l}} h_{l, i}^{2}\left\|\boldsymbol{P}_{l}^{i} \mathscr{E}_{l}^{i-1} \boldsymbol{E}_{l-1} \boldsymbol{v}\right\|_{\hat{\boldsymbol{a}}}^{2},
\end{aligned}
$$

which together with (4.37) implies that for sufficiently small $h_{0}$ there holds

$$
\begin{aligned}
\sum_{l=1}^{L} \sum_{i=1}^{N_{l}+M_{l}} \hat{\boldsymbol{a}}\left(\boldsymbol{P}_{l}^{i} \mathscr{E}_{l}^{i-1} \boldsymbol{E}_{l-1} \boldsymbol{v}, \boldsymbol{P}_{l}^{i} \mathscr{E}_{l}^{i-1} \boldsymbol{E}_{l-1} \boldsymbol{v}\right) \leq & 2 C \sum_{l=1}^{L} \hat{\boldsymbol{a}}\left(\boldsymbol{T}_{l} \boldsymbol{E}_{l-1} \boldsymbol{v}, \boldsymbol{E}_{l-1} \boldsymbol{v}\right)-C \sum_{l=1}^{L} \hat{\boldsymbol{a}}\left(\boldsymbol{T}_{l} \boldsymbol{E}_{l-1} \boldsymbol{v}, \boldsymbol{T}_{l} \boldsymbol{E}_{l-1} \boldsymbol{v}\right) \\
& +C_{12}\left(h_{0}^{2} \hat{\boldsymbol{a}}(\boldsymbol{v}, \boldsymbol{v})+h_{0}^{2} \hat{\boldsymbol{a}}\left(\boldsymbol{P}_{0} \boldsymbol{v}, \boldsymbol{P}_{0} \boldsymbol{v}\right)\right)
\end{aligned}
$$

Hence, due to (4.34)

$$
\begin{aligned}
\sum_{l=1}^{L} \hat{\boldsymbol{a}}\left(\boldsymbol{T}_{l} \boldsymbol{E}_{l-1} \boldsymbol{v}, \boldsymbol{T}_{l} \boldsymbol{E}_{l-1} \boldsymbol{v}\right) \leq & 2 C \sum_{l=1}^{L} \hat{\boldsymbol{a}}\left(\boldsymbol{T}_{l} \boldsymbol{E}_{l-1} \boldsymbol{v}, \boldsymbol{E}_{l-1} \boldsymbol{v}\right)-C \sum_{l=1}^{L} \hat{\boldsymbol{a}}\left(\boldsymbol{T}_{l} \boldsymbol{E}_{l-1} \boldsymbol{v}, \boldsymbol{T}_{l} \boldsymbol{E}_{l-1} \boldsymbol{v}\right) \\
& +C_{13}\left(h_{0}^{2} \hat{\boldsymbol{a}}(\boldsymbol{v}, \boldsymbol{v})+h_{0}^{2} \hat{\boldsymbol{a}}\left(\boldsymbol{P}_{0} \boldsymbol{v}, \boldsymbol{P}_{0} \boldsymbol{v}\right)\right),
\end{aligned}
$$

which can be written as follows

$$
\begin{aligned}
\sum_{l=1}^{L} \hat{\boldsymbol{a}}\left(\boldsymbol{T}_{l} \boldsymbol{E}_{l-1} \boldsymbol{v}, \boldsymbol{T}_{l} \boldsymbol{E}_{l-1} \boldsymbol{v}\right) \leq & \frac{2 C}{1+C} \sum_{l=1}^{L} \hat{\boldsymbol{a}}\left(\boldsymbol{T}_{l} \boldsymbol{E}_{l-1} \boldsymbol{v}, \boldsymbol{E}_{l-1} \boldsymbol{v}\right) \\
& +\frac{C_{13}}{1+C}\left(h_{0}^{2} \hat{\boldsymbol{a}}(\boldsymbol{v}, \boldsymbol{v})+h_{0}^{2} \hat{\boldsymbol{a}}\left(\boldsymbol{P}_{0} \boldsymbol{v}, \boldsymbol{P}_{0} \boldsymbol{v}\right)\right) .
\end{aligned}
$$

Taking (4.18) into account and setting $\omega:=\max \left\{\frac{2 C}{1+C}, \frac{4}{3}\left(\frac{C_{13} h_{0}^{2}}{1+C}+1\right)\right\}$, it follows that $\omega \in(0,2)$ for sufficiently small $h_{0}$.

\subsubsection{Verification of (A3)}

An application of the global strengthened Cauchy-Schwarz inequality (3.3) and arguing as in Lemma 4.5 and Lemma 4.6, we can easily prove (A3).

Lemma 4.7. Let $\boldsymbol{R}_{l}$ be given by (2.12). For sufficiently small $h_{0}$, (A3) holds true with positive constants $C_{3}$, $C_{4}$, and $C_{5}$ that only depend on the shape regularity of the meshes, the wave number $\kappa$, and the initial mesh size $h_{0}$. 
Proof. Using (3.3), we obtain

$$
\begin{aligned}
\sum_{l=0}^{L} \sum_{k=0}^{l-1} \hat{\boldsymbol{a}}\left(\boldsymbol{T}_{l} \boldsymbol{v}, \boldsymbol{T}_{k} \boldsymbol{E}_{k-1} \boldsymbol{v}\right) \leq & C\left(\sum_{l=1}^{L} \sum_{i=1}^{N_{l}+M_{l}} \hat{\boldsymbol{a}}\left(\boldsymbol{P}_{l}^{i} \mathscr{E}_{l}^{i-1} \boldsymbol{v}, \boldsymbol{P}_{l}^{i} \mathscr{E}_{l}^{i-1} \boldsymbol{v}\right)+\hat{\boldsymbol{a}}\left(\boldsymbol{P}_{0} \boldsymbol{v}, \boldsymbol{P}_{0} \boldsymbol{v}\right)\right)^{\frac{1}{2}} \\
& \cdot\left(\sum_{l=1}^{L} \sum_{i=1}^{N_{l}+M_{l}} \hat{\boldsymbol{a}}\left(\boldsymbol{P}_{l}^{i} \mathscr{E}_{l}^{i-1} \boldsymbol{E}_{l-1} \boldsymbol{v}, \boldsymbol{P}_{l}^{i} \mathscr{E}_{l}^{i-1} \boldsymbol{E}_{l-1} \boldsymbol{v}\right)+\hat{\boldsymbol{a}}\left(\boldsymbol{P}_{0} \boldsymbol{v}, \boldsymbol{P}_{0} \boldsymbol{v}\right)\right)^{\frac{1}{2}}
\end{aligned}
$$

The assertion follows directly from (4.18), (4.33) and (4.38).

\section{Numerical Results}

We illustrate the theoretical convergence results and test the performance of LMM by the results of two numerical examples. For the solution of the algebraic systems resulting from the curl-conforming edge element discretization of the time-harmonic Maxwell equation, LMM (Algorithm 2.1) is used as a preconditioner for GMRES (PGMRES). The adaptive mesh refinement has been done by Dörfler marking [17] on the basis of the residual-type a posteriori error estimators from $[14,42]$, and the refinement itself has been realized by the newest vertex bisection algorithm. Since the computations at the $l$-th level involve only local nodes corresponding to the components in $\widetilde{\mathcal{E}}_{l}$ and $\widetilde{\mathcal{N}}_{l}$, the computational cost of the local multigrid algorithm is proportional to the number of degrees of freedom (DOF).

At the $l$-th level, the discrete problem reads $\mathbb{A}_{l} \mathbf{u}_{l}=\mathbf{F}_{l}$. We denote by $\mathbf{u}_{l}^{0}$ the prolongated coarse grid correction, i.e., $\mathbf{u}_{l}^{0}=\mathbf{I}_{l-1} \mathbf{u}_{l-1}$ where $\mathbf{I}_{l-1}$ is the transfer matrix realizing the prolongation from the coarse to the fine edge element space. We further refer to $\mathbf{r}_{l}^{n}=\mathbf{F}_{l}-\mathbb{A}_{l} \mathbf{u}_{l}^{n}$ as the residual with respect to the $n$-th iteration. The PGMRES algorithm terminates when

$$
\left\|\mathbf{r}_{l}^{n}\right\| /\left\|\mathbf{r}_{l}^{0}\right\| \leq 10^{-6}
$$

where $\|\cdot\|$ stands for the Euclidean norm. The number of iteration steps required to achieve the desired accuracy is denoted by iter.

The theoretical results obtained in the previous section predict an initial mesh size $h_{0}$ satisfying $\kappa^{2} h_{0}^{s} \leq C$. As will be seen in the following examples, in actual computations, however, $h_{0}$ can be chosen slightly coarser than predicted by theory.

Example 5.1. We consider the time-harmonic Maxwell equation (1.1a), (1.1b) on the L-shaped domain

$$
\begin{aligned}
& \Omega=(-1,1) \times(-1,1) \times(0,1) \backslash\left(\Omega_{1} \cup \Omega_{2} \cup \Omega_{3}\right), \quad \Omega_{1}=(-1,0) \times(-1,0) \times(0,1), \\
& \Omega_{2}=(-1,-0.5) \times(0,0.5) \times(0,1), \quad \Omega_{3}=(0,0.5) \times(-1,-0.5) \times(0,1),
\end{aligned}
$$

and choose the right-hand side $\boldsymbol{f}$ according to $\boldsymbol{f}=(1,1,1)^{T}$.

We test the cases $\kappa^{2}=9$ and $\kappa^{2}=100$. The scaling factor is chosen as $\gamma=0.6$ in the local Hiptmair-Jacobi smoother. For $\kappa^{2}=9$, the restriction $\kappa^{2} h_{0}^{s} \leq C$ requires $h_{0} \approx 0.01 \sim 0.1$ in theory, but $h_{0} \approx 0.5$ worked well in our experiments. Figure 2 (left) shows the locally refined mesh with 249231 DOF at the 21st refinement level, whereas Figure 2 (right) displays the associated discrete solution vector on the boundary. We observe that the singularity of the solution is near the corner lines $l_{1}: x_{1}=x_{2}=0, l_{2}: x_{1}=-0.5, x_{2}=0.5$ and $l_{3}: x_{1}=0.5, x_{2}=-0.5$. Table 1 shows that the iteration steps of PGMRES with the local Hiptmair-Jacobi smoother or the local Hiptmair-Gauss-Seidel smoother are almost uniform and bounded independently of mesh sizes and mesh levels. We find that even for the finer initial mesh with $h_{0} \approx 0.25$, the convergence properties of PGMRES with the two types of local smoothers are almost the same as for $h_{0} \approx 0.5$.

For $\kappa^{2}=100$, we have chosen $h_{0} \approx 0.16$ which is also coarser than predicted by theory. Table 1 shows that in case of this initial mesh size LMM is indeed a good preconditioner for GMRES. 

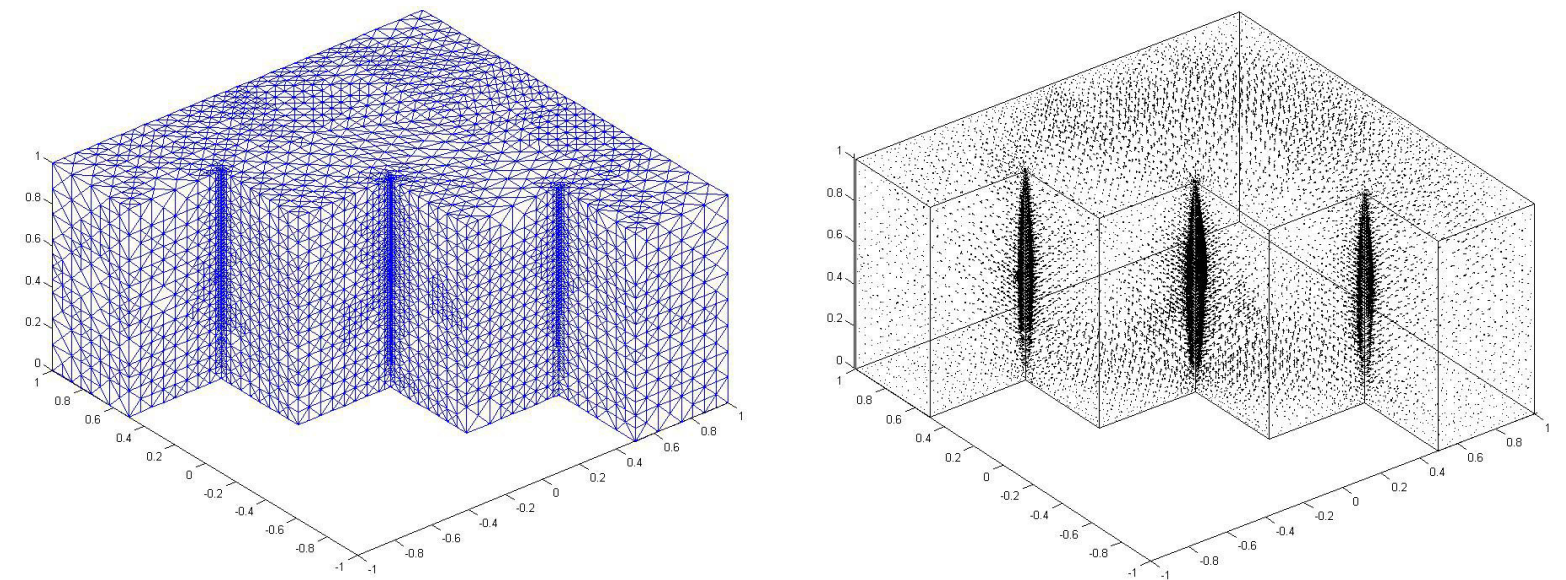

Figure 2. Example $5.1\left(\kappa^{2}=9\right)$ : locally refined mesh (left) with 249231 DOF and the discrete solution vector (right) on the boundary at the 21 st refinement level.

TABLE 1. Example 5.1: the number of PGMRES iterations with the local Hiptmair-Jacobi smoother and the local Hiptmair-Gauss-Seidel smoother for $\kappa^{2}=9$ and $\kappa^{2}=100$.

\begin{tabular}{|r|c|c|c|c|c|c|c|}
\hline \multicolumn{4}{|c|}{$\kappa^{2}=9$} & \multicolumn{4}{c|}{$\kappa^{2}=100$} \\
\cline { 3 - 4 } Level & \multirow{2}{*}{ DOF } & \multicolumn{2}{|c|}{ Iter } & \multirow{2}{*}{ Level } & \multirow{2}{*}{ DOF } & \multicolumn{2}{|c|}{ Iter } \\
\cline { 3 - 4 } & & GS & Jacobi & & & GS & Jacobi \\
\hline 13 & 21911 & 12 & 19 & 5 & 37803 & 19 & 27 \\
15 & 40893 & 13 & 20 & 7 & 74471 & 23 & 34 \\
17 & 75948 & 12 & 19 & 9 & 125880 & 27 & 42 \\
19 & 138427 & 12 & 19 & 11 & 174715 & 26 & 39 \\
21 & 249231 & 12 & 19 & 13 & 298580 & 27 & 42 \\
23 & 445534 & 13 & 20 & 15 & 474480 & 27 & 43 \\
25 & 768161 & 11 & 19 & 17 & 817911 & 27 & 44 \\
27 & 1342894 & 11 & 19 & 19 & 1422440 & 26 & 43 \\
\hline
\end{tabular}

Figure 3 shows that for $\kappa^{2}=9$ and $\kappa^{2}=100$ the CPU times (in seconds) of each PGMRES iteration with different types of local smoothers is almost linear in terms of DOF which, together with the almost uniform convergence, implies quasi-optimality of the PGMRES algorithm.

The next example deals with the application of LMM to the time-harmonic Maxwell equations on a non-Lipschitz domain.

Example 5.2. We test the time-harmonic problem (1-1.1b) on a non-Lipschitz domain with two cubic cavities. The computational domain reads

$$
\begin{aligned}
& \Omega=(-1,1) \times(-1,1) \times(0,2) \backslash\left(Q_{1} \bigcup Q_{2}\right), \\
& Q_{1}=(-0.4,0) \times(-0.4,0) \times(0.6,1), \quad Q_{2}=(0,0.4) \times(0,0.4) \times(1,1.4),
\end{aligned}
$$

and the right-hand side $\boldsymbol{f}$ is again given by $\boldsymbol{f}=(1,1,1)^{T}$.

We test $\kappa^{2}=1,9,25,49,100$ and only use the local Hiptmair-Gauss-Seidel smoother in this experiment. In order to illustrate the convergence property of PGMRES, we have first chosen an initial mesh of mesh size 


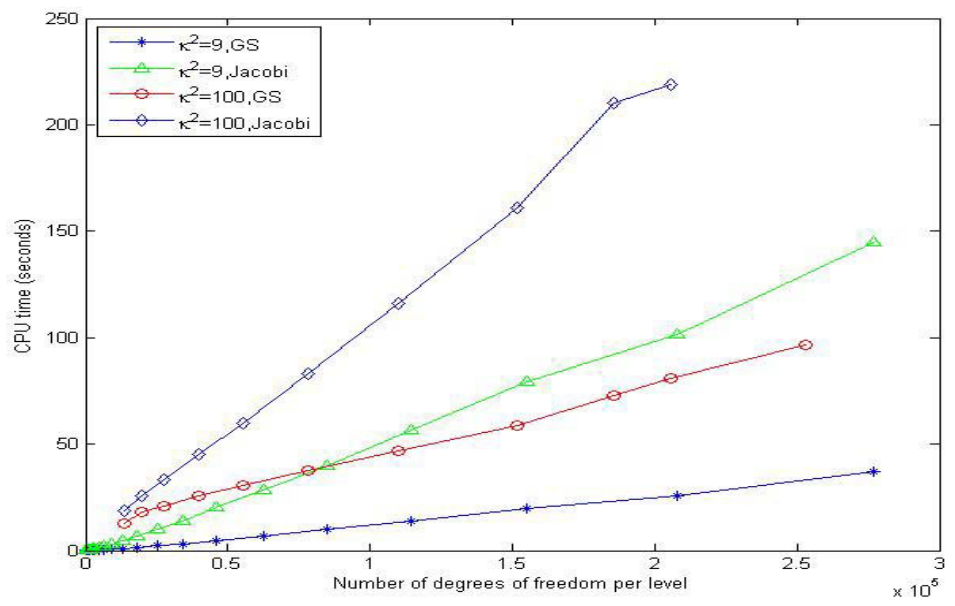

Figure 3. Example 5.1: CPU times of PGMRES iterations with different smoothers for $\kappa^{2}=9$ and $\kappa^{2}=100$.

TABLE 2. Example 5.2: the number of PGMRES iterations with the local Hiptmair-GaussSeidel smoother for $\kappa^{2}=1,9,25,49,100$ based on the same initial mesh with $h_{0} \approx 0.3$.

\begin{tabular}{|c|c|ccccccc|}
\hline \multirow{3}{*}{$\kappa^{2}=1$} & Level & 5 & 7 & 9 & 11 & 13 & 15 & 17 \\
\cline { 2 - 10 } & DOF & 20419 & 39336 & 77847 & 152017 & 288629 & 535882 & 991044 \\
\cline { 2 - 10 } & iter & 11 & 12 & 12 & 12 & 12 & 12 & 12 \\
\hline \multirow{3}{*}{$\kappa^{2}=9$} & Level & 5 & 7 & 9 & 11 & 13 & 15 & 17 \\
\cline { 2 - 10 } & DOF & 16935 & 33359 & 66451 & 127286 & 240873 & 450916 & 821193 \\
\cline { 2 - 9 } & iter & 12 & 12 & 13 & 13 & 13 & 13 & 12 \\
\hline \multirow{3}{*}{$\kappa^{2}=25$} & Level & 5 & 7 & 9 & 11 & 13 & 15 & 17 \\
\cline { 2 - 9 } & DOF & 18042 & 34848 & 69961 & 137471 & 262696 & 487616 & 873067 \\
\cline { 2 - 9 } & iter & 15 & 16 & 17 & 18 & 18 & 18 & 18 \\
\hline \multirow{3}{*}{$\kappa^{2}=49$} & Level & 6 & 8 & 10 & 12 & 14 & 16 & 18 \\
\cline { 2 - 9 } & DOF & 19546 & 37918 & 73673 & 124783 & 229763 & 422671 & 756569 \\
\cline { 2 - 9 } & iter & 28 & 31 & 34 & 34 & 33 & 34 & 34 \\
\hline \multirow{3}{*}{$\kappa^{2}=100$} & Level & 7 & 9 & 11 & 15 & 19 & 23 & 27 \\
\cline { 2 - 9 } & DOF & 18548 & 27041 & 38480 & 83130 & 148381 & 304108 & 857085 \\
\cline { 2 - 9 } & iter & 128 & 149 & 165 & 169 & 154 & 153 & 150 \\
\hline
\end{tabular}

$h_{0} \approx 0.3$. Figure 4 (left) shows a part of the locally refined mesh with 331369 DOF on the boundary for $\kappa^{2}=9$. We observe that the mesh is always locally refined near the boundary of the cavities. Figure 4 (right) displays the associated discrete solution vector on the boundary. Figure 5 shows that for each wave number the CPU time of the PGMRES iteration is almost linear in terms of DOF. Table 2 indicates that in each case the iteration steps remain almost uniform on different levels.

For $\kappa^{2}=100$, the approximation is far from the true solution, if the initial mesh size is too coarse. Indeed, we observe that for this higher wave number the convergence of PGMRES is much better for the finer initial mesh. This is reflected by the results displayed in Tables 3 and 4 . 

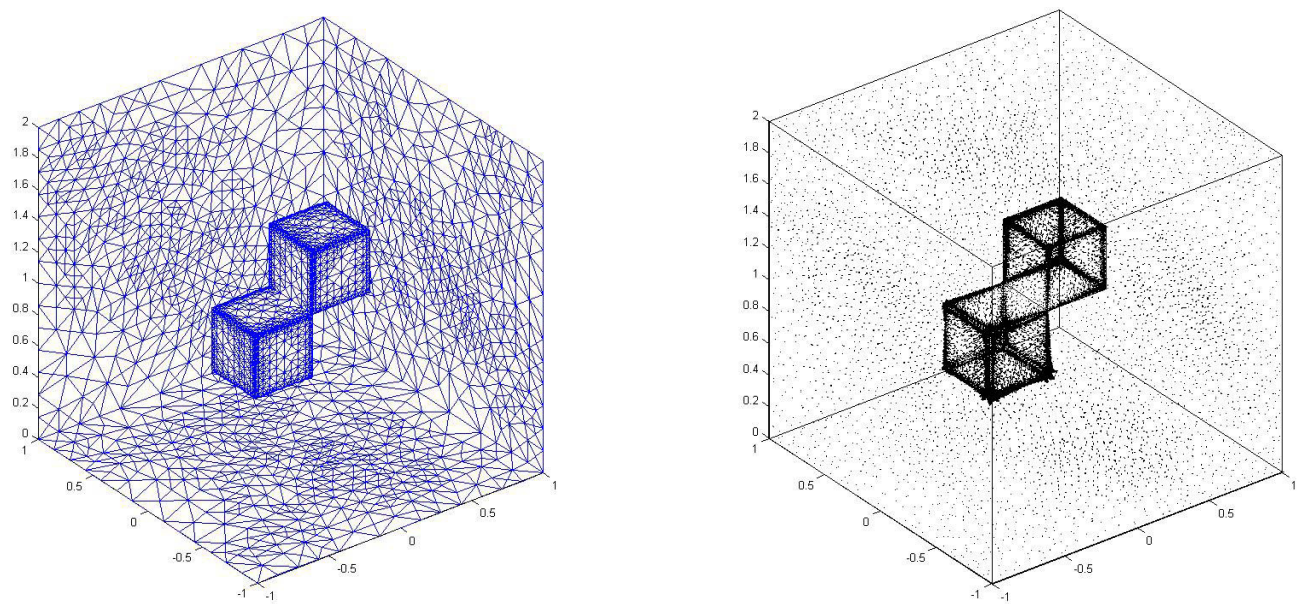

Figure 4. Example $5.2\left(\kappa^{2}=9\right)$ : Locally refined mesh (left, part of surface mesh on the boundary) with $331369 \mathrm{DOF}$, and the discrete solution vector (right) on the boundary at the 14-th refinement level.

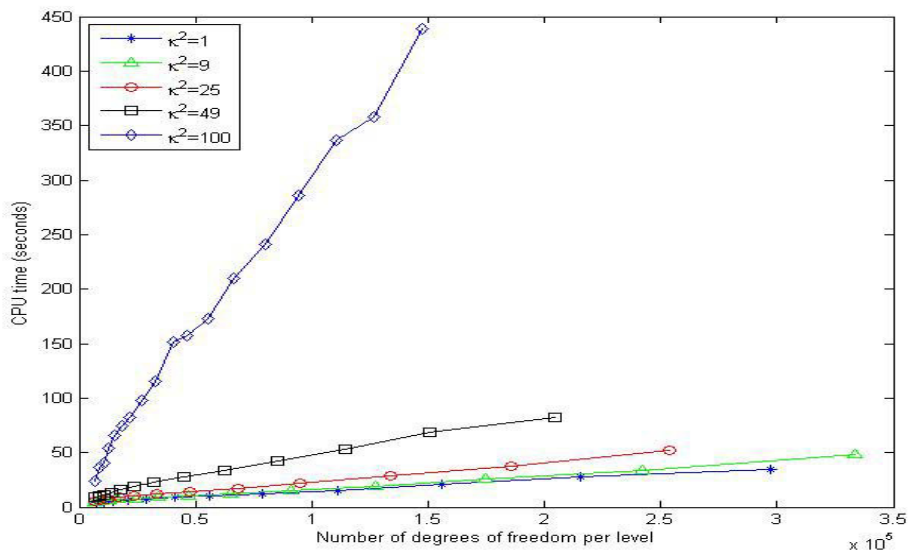

Figure 5. Example 5.2: CPU times of PGMRES iterations with the local Hiptmair-GaussSeidel smoother for $\kappa^{2}=1,9,25,49,100$.

TABLE 3. Example 5.2: the number of PGMRES iterations for $\kappa^{2}=100$ based on different initial meshes.

\begin{tabular}{|c|c|ccccccc|}
\hline \multirow{3}{*}{$h_{0} \approx 0.25$} & Level & 8 & 10 & 12 & 15 & 18 & 21 & 24 \\
\cline { 2 - 10 } & DOF & 36866 & 54596 & 78258 & 138683 & 254987 & 514008 & 876407 \\
\cline { 2 - 9 } & Iter & 104 & 108 & 108 & 111 & 107 & 112 & 111 \\
\hline \multirow{3}{*}{$h_{0} \approx 0.1$} & Level & 8 & 10 & 12 & 14 & 17 & 20 & 23 \\
\cline { 2 - 9 } & DOF & 259677 & 324188 & 392348 & 481689 & 613173 & 740370 & 951532 \\
\cline { 2 - 9 } & Iter & 16 & 18 & 20 & 22 & 28 & 28 & 28 \\
\hline
\end{tabular}


Acknowledgements. We are indebted to Haijun Wu (Nanjing University, China) for his most valuable assistance regarding the implementation of LMM for the edge element discretized time-harmonic Maxwell equation.

\section{REFERENCES}

[1] B. Aksoylu and M. Holst, Optimality of multilevel preconditioners for local mesh refinement in three dimensions. SIAM J. Numer. Anal. 44 (2006) 1005-1025.

[2] B. Aksoylu, S. Bond and M. Holst, An odyssey into local refinement and multilevel preconditioning III: implementation and numerical experiments. SIAM J. Sci. Comput. 25 (2003) 478-498.

[3] D. Arnold, R. Falk and R. Winther, Multigrid in H(div) and H(curl). Numer. Math. 85 (2000) 197-218.

[4] D. Bai and A. Brandt, Local mesh refinement multilevel techniques. SIAM J. Sci. Stat. Comput. 8 (1987) $109-134$.

[5] E. Bänsch, Local mesh refinement in 2 and 3 dimensions. Impact Comput. Sci. Eng. 3 (1991) 181-191.

[6] R. Beck, P. Deufhard, R. Hiptmair, R.H.W. Hoppe and B. Wohlmuth, Adaptive multilevel methods for edge element discretizations of Maxwell's equations. Surv. Math. Indust. 8 (1999) 271-312.

[7] R. Beck, R. Hiptmair, R.H.W. Hoppe and B. Wohlmuth, Residual based a posteriori error estimators for eddy current computation. ESAIM: M2AN 34 (2000) 159-182.

[8] A. Bossavit, Computational Electromagnetism: Variational Formulations, Complementarity, Edge Elements. Academic Press, San Diego (1998).

[9] J.H. Bramble, Multigrid Methods. Pitman (1993).

[10] J.H. Bramble, J.E. Pasciak, J. Wang and J. Xu, Convergence estimates for product iterative methods with applications to domain decomposition. Math. Comp. 57 (1991) 23-45.

[11] J.H. Bramble, D.Y. Kwak and J.E. Pasciak, Uniform convergence of multigrid V-cycle iterations for indefinite and nonsymmetric problems. SIAM J. Numer. Anal. 31 (1994) 1746-1763.

[12] C. Carstensen and R.H.W. Hoppe, Convergence analysis of an adaptive edge finite element method for the $2 \mathrm{~d}$ eddy current equations. J. Numer. Math. 13 (2005) 19-32.

[13] H. Chen and $\mathrm{X}$. Xu, Local multilevel methods for adaptive finite element methods for nonsymmetric and indefinite elliptic boundary value problems. SIAM J. Numer. Anal. 47 (2010) 4492-4516.

[14] Z. Chen, L. Wang and W. Zheng, An adaptive multilevel method for time-harmonic Maxwell equations with singularities. SIAM J. Sci. Comput. 29 (2007) 118-138.

[15] J. Chen, Y. Xu and J. Zou, Convergence analysis of an adaptive edge element method for Maxwell's equations. Appl. Numer. Math. 59 (2009) 2950-2969.

[16] W. Dahmen and A. Kunoth, Multilevel preconditioning. Numer. Math. 63 (1992) 315-344.

[17] W. Dörfler, A convergent adaptive algorithm for Poisson's equation. SIAM J. Numer. Anal. 33 (1996) $1106-1124$.

[18] J. Gopalakrishnan and J. Pasciak, Overlapping Schwarz preconditioners for indefinite time harmonic Maxwell equations. Math. Comp. 72 (2003) 1-15.

[19] J. Gopalakrishnan, J. Pasciak and L.F. Demkowicz, Analysis of a multigrid algorithm for time harmonic Maxwell equations. SIAM J. Numer. Anal. 42 (2004) 90-108.

[20] R. Hiptmair, Multigrid method for Maxwell's equations. SIAM J. Numer. Anal. 36 (1998) 204-225.

[21] R. Hiptmair, Finite elements in computational electromagnetism. Acta Numer. 11 (2002) 237-339.

[22] R. Hiptmair and J. Xu, Nodal auxiliary spaces preconditions in $\mathrm{H}(\mathrm{curl})$ and $\mathrm{H}(\mathrm{div})$ spaces. SIAM J. Numer. Anal. 45 (2007) 2483-2509.

[23] R. Hiptmair and W. Zheng, Local multigrid in $\boldsymbol{H}(\mathbf{c u r l}, \Omega)$. J. Comput. Math. 27 (2009) 573-603.

[24] R. Hiptmair, H. Wu and W. Zheng, On uniform convergence theory of local multigrid methods in $H^{1}(\Omega)$ and $\boldsymbol{H}(\mathbf{c u r l}, \Omega)$. Preprint (2010).

[25] R.H.W. Hoppe and J. Schöberl, Convergence of adaptive edge element methods for the 3D eddy currents equations. J. Comput. Math. 27 (2009) 657-676.

[26] R.H.W. Hoppe, X. Xu and H. Chen, Local Multigrid on Adaptively Refined Meshes and Multilevel Preconditioning with Applications to Problems in Electromagnetism and Acoustics, in Efficient Preconditioned Solution Methods for Elliptic Partial Differential Equations, edited by O. Axelsson and J. Karatson. Bentham, Bussum, The Netherlands (2010) 125-145.

[27] R. Leis, Exterior boundary-value problems in mathematical physics, in Trends in Applications of Pure Mathematics to Mechanics, edited by H. Zorski. Monographs Stud. Math. 5 (1979) 187-203.

[28] P. Monk, A posteriori error indicators for Maxwell's equations. Comput. Appl. Math. 100 (1998) 173-190.

[29] P. Monk, Finite element methods for Maxwell equations, Numerical Mathematics and Scientific Computation. Oxford University Press, New York (2003).

[30] J.-C. Nédélec, Mixed finite element in $\mathbb{R}^{3}$. Numer. Math. 35 (1980) 315-341.

[31] J.-C. Nédélec, A new family of mixed finite elements in $\mathbb{R}^{3}$. Numer. Math. 50 (1986) 57-81.

[32] P. Oswald, Multilevel Finite Element Approximation: Theory and Applications. Teubner, Stuttgart (1994).

[33] U. Rüde, Fully adaptive multigrid methods. SIAM J. Numer. Anal. 30 (1993) 230-248.

[34] O. Sterz, A. Hauser and G. Wittum, Adaptive local multigrid methods for solving time-harmonic eddy current problems. IEEE Trans. Magn. 42 (2006) 309-318. 
[35] L. Tartar, Introduction to Sobolev Spaces and Interpolation Theory. Springer, Berlin, Heidelberg, New York (2007).

[36] H. Whitney, Geometric Integration Theory. Princeton University Press, Princeton (1957).

[37] H.J. Wu and Z.M. Chen, Uniform convergence of multigrid V-cycle on adaptively refined finite element meshes for second order elliptic problems. Sci. China 39 (2006) 1405-1429.

[38] J. Xu, L. Chen and R. Nochetto, Optimal multilevel methods for H(grad), H(curl), and H(div) systems on graded and unstructured grids, in Multiscale, Nonlinear and Adaptive Approximation. Springer (2009) 599-659.

[39] X. Xu, H. Chen and R.H.W. Hoppe, Optimality of local multilevel methods on adaptively refined meshes for elliptic boundary value problems. J. Numer. Math. 18 (2010) 59-90.

[40] X. Xu, H. Chen and R.H.W. Hoppe, Optimality of local multilevel methods for adaptive nonconforming P1 finite element methods. J. Comput. Math. (2012), in press.

[41] L. Zhong, L. Chen and J. Xu, Convergence of adaptive edge finite element methods for H(curl)-elliptic problems. Numer. Lin. Algebra Appl. 17 (2009) 415-432.

[42] L. Zhong, L. Chen, S. Shu, G. Wittum and J. Xu, Quasi-optimal convergence of adaptive edge finite element methods for three dimensional indefinite time-harmonic Maxwell's equations. Math. Comp. 81 (2012), 623-642. 